\title{
A Novel Algorithm for Power Flow Transferring Identification Based on WAMS
}

\author{
Xu Yan, ${ }^{1}$ Han Ping, ${ }^{1}$ and Zhang $\mathrm{Li}^{2}$ \\ ${ }^{1}$ State Key Laboratory of Alternate Electrical Power System with Renewable Energy Source, North China Electric Power University, \\ Baoding 071000, China \\ ${ }^{2}$ School of Electrical and Electronic Engineering, North China Electric Power University, Baoding 071000, China
}

Correspondence should be addressed to Han Ping; shdapple@sina.com

Received 4 May 2015; Accepted 14 September 2015

Academic Editor: Yang Tang

Copyright (C) $2015 \mathrm{Xu}$ Yan et al. This is an open access article distributed under the Creative Commons Attribution License, which permits unrestricted use, distribution, and reproduction in any medium, provided the original work is properly cited.

\begin{abstract}
After a faulted transmission line is removed, power flow on it will be transferred to other lines in the network. If those lines are heavily loaded beforehand, the transferred flow may cause the nonfault overload and the incorrect operation of far-ranging backup relays, which are considered as the key factors leading to cascading trips. In this paper, a novel algorithm for power flow transferring identification based on wide area measurement system (WAMS) is proposed, through which the possible incorrect tripping of backup relays will be blocked in time. A new concept of Transferred Flow Characteristic Ratio (TFCR) is presented and is applied to the identification criteria. Mathematical derivation of TFCR is carried out in detail by utilization of power system short circuit fault modeling. The feasibility and effectiveness of the proposed algorithm to prevent the malfunction of backup relays are demonstrated by a large number of simulations.
\end{abstract}

\section{Introduction}

With the interconnection of power networks, transmission lines are operating close to their limit. Power system relay protection is of great importance to the security and the stability of power system. Recently, many well-known blackouts occurred worldwide [1-5]. Related researches show that the incorrect operation of far-ranging backup relays is relevant to most of them. After the faulted transmission line is tripped by protective relays, the flow on it will be transferred to other lines, which will result in overloads on them if they have been heavily loaded before. Although the backup relays such as zone 3 distance relays will remove these lines according to their setting principles, they will deteriorate the system status and will promote the process of system collapse. If the transferred power flow is identified, the backup relays tripping will be blocked in time and some measures to eliminate the overload will be taken to prevent cascading trips. Unfortunately, because of the only utilization of local data to make judgment, the existing traditional backup protection cannot distinguish whether the malfunction is caused by internal fault or by transferred power flow.

The rise of wide area measurement system (WAMS) has opened a new gate for power system wide area backup protection [6-9]. The system operating parameters, such as three-phase voltages and currents, power angle, and active and reactive power flows, can be updated every 20-50 milliseconds; thus it is allowed to acquire and to deal with the synchronous data of the whole system thanks to the time delay of backup protections. Although the substitution of supervisory control and data acquisition (SCADA) system by WAMS is limited by the current technical and economic conditions $[10,11]$, the complete observability of power system can be fully guaranteed according to the optimal placement methods of the limited-number GPS-based synchronized phasor measurement units (PMUs). Therefore, the realization of real-time tracking of the complicated network topology makes it possible to carry out researches on backup protection scheme taking the whole system safe, stable, and reliable operation as targets $[12,13]$. 
The past decades have witnessed some categories of methods provided by a growing number of investigators at home and abroad to prevent cascading trips, such as multipoint measurement information based differential backup protection $[14,15]$, adaptive scheme for distance protection [16], expert decision system for wide area backup protection of transmission networks $[17,18]$, and fault directional comparison principle [19]. However, these references focus on the optimal procedure of power system fault elimination and how to avoid the incorrect operation of protective relays by internal fault, not taking prevention of backup relays tripping caused by nonfault overload into consideration. On the other hand, the flow transferring relativity factor in the paper [20] and the DC flow based transfer power flow sensitivity factors in the paper [21] are, respectively, presented to estimate the postfault power flow distribution (the branch current and the active power flow distribution). By comparing with the measured flow by WAMS, whether the overload is caused by the transferred flow or not can be identified. However, these methods may identify incorrectly because the variation in reactive power injection caused by fault elimination is ignored during the estimation of postfault flow distribution. Besides, with the number of grid nodes becoming larger, the computational burden of these methods is too heavy to estimate the flow distribution in the allowed delay of the backup protection.

In this paper, a novel algorithm for power flow transferring identification based on WAMS is proposed, through which transmission line overload caused by the transferred power flow will be identified exactly, and the possible incorrect tripping of backup relays will be blocked in time. The original property of the distance protection that protective relays operate as soon as the delay time is out will not be changed. The novel identification criteria of the algorithm employ a newly proposed concept of Transferred Flow Characteristic Ratio (TFCR) composed of 3 ratios which are calculated after zone 3 distance protection starts. Because the variation in reactive power infection, the load type, the different fault locations, and transition resistances do not influence the identification accuracy, the proposed algorithm owns a high reliability. Besides, the settings of the identification criteria can be determined in terms of the analysis in this paper as well as the on-the-spot conditions, making the proposed algorithm highly flexible. Moreover, as the algorithm is simple in principle and the electrical quantities required for the TFCR can be directly measured by PMUs, the burdens of computation and communication are lightened to improve the performance of traditional backup protection.

The rest of this paper is structured as follows. In Section 2, two ratios of TFCR are derived mathematically by utilization of the fault current model. Two current criteria to identify internal fault or nonfault overload caused by the transferred power flow are proposed. Section 3 solves another ratio of TFCR using the fault voltage model and proposes the voltage criterion. Section 4 states the logical relationship of the 3 criteria and provides the scheme and its implementation of the novel algorithm for power flow transferring identification. A number of simulation results in Section 5 validate

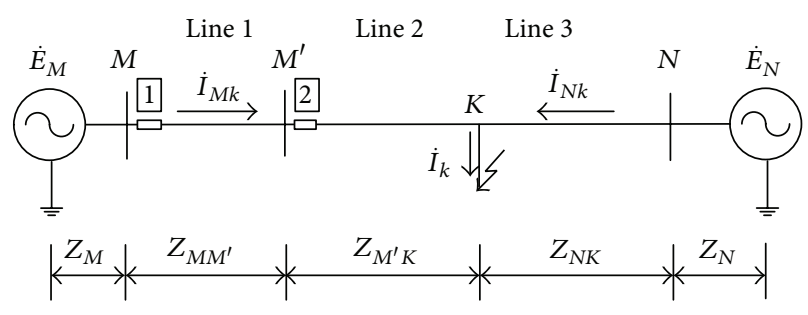

FIgURE 1: Power system.

the effectiveness of the proposed algorithm. The paper is concluded in Section 6.

\section{Mathematical Derivation of Criteria Based on Fault Current Model}

If the overload is caused by the transferred power flow, threephase currents on transmission lines will stay symmetric and the three-phase current increments will have equal amplitudes. Moreover, zero-sequence current will not exist. However, if the overload resulted from an asymmetric short circuit fault, the three-phase current increments which are called fault components of 3 phase currents will not be equal. Furthermore, if it is an earth fault, there will appear the zero-sequence current. In order to establish the criteria to identify the asymmetric short circuit fault, two characteristic ratios of a transmission line calculated by fault components of 3 phase currents and zero-sequence current are derived mathematically via current distribution factor theory and asymmetric short circuit fault modeling.

2.1. Theory of Current Distribution Factor. Current distribution factor represents the proportion of fault current supplied by each power source in the network. The definition of current distribution factors and their usage in solving fault components of three phase currents are explained in the following paragraph.

As is shown in Figure 1, assume that a short circuit fault occurs at point $K$. Distance Protection 1 is installed near Bus $M$, of which zone 3 is considered as the far-ranging backup protection of Distance Protection 2 installed near Bus $M^{\prime}$. We select phase $A$ as the reference phase; that is to say, we use sequence components of phase $A$ to solve three-phase voltage phasors and current phasors during a fault. The $A$ phase current flowing out of fault point $K$ is represented by $\dot{I}_{k}$ and the fault component of $A$-phase current on Line 1 is represented by $\dot{I}_{M k}$.

Regard the sequence impedances of each line as the known quantities. The positive-sequence network based on the power system in Figure 1 can be converted to the equivalent network shown in Figure 2.

According to Figure 2, $Z_{S M 1}=Z_{M 1}+Z_{M M^{\prime} 1}+Z_{M^{\prime} K 1}$ and $Z_{S N 1}=Z_{N 1}+Z_{N K 1}$. The positive-sequence components of $\dot{I}_{k}$ and $\dot{I}_{M k}$ are represented by $\dot{I}_{k 1}$ and $\dot{I}_{M k 1}$, respectively. 


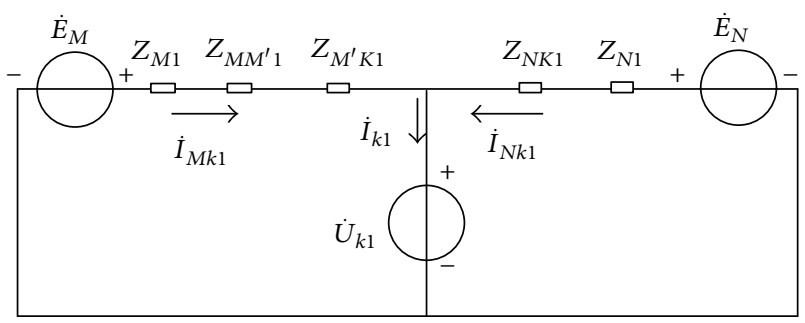

FIGURE 2: Equivalent positive-sequence network.

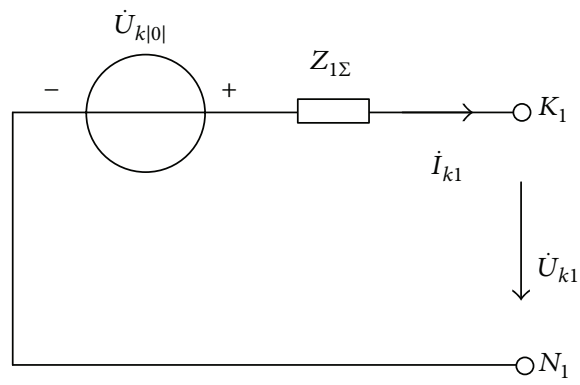

FIgURE 3: Simplified positive-sequence network.

The positive-sequence current distribution factor $C_{M 1}$ can be defined as follows:

$$
C_{M 1}=\frac{\dot{I}_{M k 1}}{\dot{I}_{k 1}}=\frac{\dot{I}_{k 1} Z_{1 \Sigma} / Z_{S M 1}}{\dot{I}_{k 1}}=\frac{Z_{1 \Sigma}}{Z_{S M 1}},
$$

where $Z_{1 \Sigma}=\left(Z_{S M 1} \cdot Z_{S N 1}\right) /\left(Z_{S M 1}+Z_{S N 1}\right)$.

Particularly, $Z_{1 \Sigma}$ represents the equivalent positivesequence impedance to the fault point. Figure 3 shows the simplified positive-sequence network, of which $\dot{U}_{k|0|}$ represents the Thevenin equivalent potential to the fault point. It will be used to solve the fault component of branch current during two-phase grounding fault in the next subsection.

Figure 4 shows the negative-sequence network based on Figure 1, in which the negative-sequence components of $\dot{I}_{k}$ and $\dot{I}_{M k}$ are represented by $\dot{I}_{k 2}$ and $\dot{I}_{M k 2}$. The zero-sequence components of $\dot{I}_{k}$ and $\dot{I}_{M k}$ are represented by $\dot{I}_{k 0}$ and $\dot{I}_{M k 0}$ in the zero-sequence network of Figure 5.

In Figure 4, there are $Z_{S M 2}=Z_{M 2}+Z_{M M^{\prime} 2}+Z_{M^{\prime} K 2}$ and $Z_{S N 2}=Z_{N 2}+Z_{N K 2}$. Similarly in Figure 5 , there are $Z_{S M 0}=$ $Z_{M 0}+Z_{M M^{\prime}}+Z_{M^{\prime} K 0}$ and $Z_{S N 0}=Z_{N 0}+Z_{N K 0}$. The negativesequence and zero-sequence current distribution factors $C_{M 2}$ and $C_{M 0}$ can be defined as follows:

$$
\begin{gathered}
C_{M 2}=\frac{\dot{I}_{M k 2}}{\dot{I}_{k 2}}=\frac{\dot{I}_{k 2} Z_{2 \Sigma} / Z_{S M 2}}{\dot{I}_{k 2}}=\frac{Z_{2 \Sigma}}{Z_{S M 2}}, \\
C_{M 0}=\frac{\dot{I}_{M k 0}}{\dot{I}_{k 0}}=\frac{\dot{I}_{k 0} Z_{0 \Sigma} / Z_{S M 0}}{\dot{I}_{k 0}}=\frac{Z_{0 \Sigma}}{Z_{S M 0}},
\end{gathered}
$$

where $Z_{2 \Sigma}=\left(Z_{S M 2} \cdot Z_{S N 2}\right) /\left(Z_{S M 2}+Z_{S N 2}\right)$ and $Z_{0 \Sigma}=\left(Z_{S M 0}\right.$. $\left.Z_{S N 0}\right) /\left(Z_{S M 0}+Z_{S N 0}\right)$.

Obviously, $Z_{2 \Sigma}$ and $Z_{0 \Sigma}$ represent the equivalent negativesequence and zero-sequence impedances to the fault point. Figures 6 and 7 show the simplified negative-sequence and

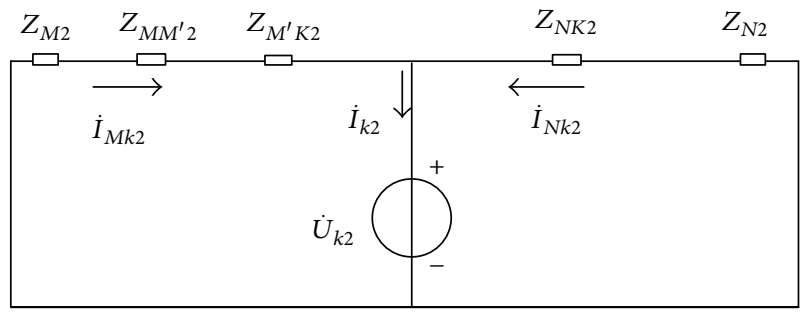

FIGURE 4: Negative-sequence network.

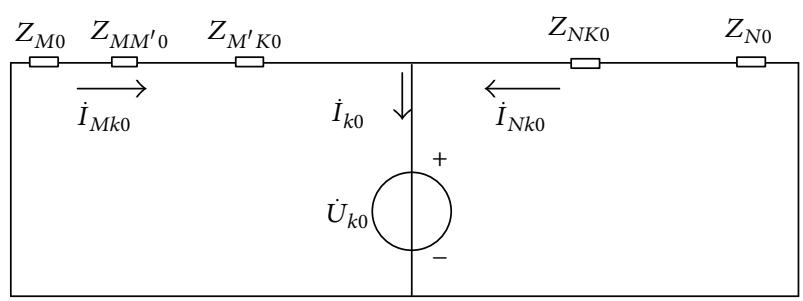

Figure 5: Zero-sequence network.

zero-sequence networks, which will also be used in the next subsection.

To deal with power network with multiple branches, the utilization of sequence networks like Figures 2, 4, and 5 will bring great convenience for acquisition of current distribution factors. Equations (1)-(3) demonstrate that current distribution factors depend on structure and parameters of network and distance to fault, having nothing to do with variation in electromotive force and types of fault. Thus it is convenient to estimate $\dot{I}_{M k 1}, \dot{I}_{M k 2}$, and $\dot{I}_{M k 0}$ by utilizing current distribution factors acquired after a fault occurs.

As a general rule, we consider that the fault point $K$ is far from the electric generators. Since in high-voltage power system the impedance of generator is far smaller than that of transmission line, the sequence components of $Z_{M}$ and $Z_{N}$ are too small to be involved in (1) and (2). For a transmission line, as the positive-sequence impedance is considered to be equal to the negative-sequence impedance, that is to say, $Z_{S M 2}=Z_{S M 1}$ and $Z_{2 \Sigma}=Z_{1 \Sigma}$, therefore $C_{M 2}=C_{M 1}$. Equations (1)-(3) are rewritten as follows:

$$
\begin{aligned}
& \dot{I}_{M k 1}=C_{M 1} \dot{I}_{k 1}, \\
& \dot{I}_{M k 2}=C_{M 2} \dot{I}_{k 2}=C_{M 1} \dot{I}_{k 2}, \\
& \dot{I}_{M k 0}=C_{M 0} \dot{I}_{k 0} .
\end{aligned}
$$

Suppose that the fault components of 3 phase currents at the location of Protection 1 in Figure 1 are represented by $\dot{I}_{M k a}, \dot{I}_{M k b}$, and $\dot{I}_{M k c}$. Obviously, $\dot{I}_{M k a}=\dot{I}_{M k}$. According to symmetrical component method, we can use sequence components of $\dot{I}_{M k}$ represented by $\dot{I}_{M k 1}, \dot{I}_{M k 2}$, and $\dot{I}_{M k 0}$ to estimate $\dot{I}_{M k a}, \dot{I}_{M k b}$, and $\dot{I}_{M k c}$ through

$$
\left[\begin{array}{l}
\dot{I}_{M k a} \\
\dot{I}_{M k b} \\
\dot{I}_{M k c}
\end{array}\right]=\left[\begin{array}{l}
\dot{I}_{M k} \\
\dot{I}_{M k b} \\
\dot{I}_{M k c}
\end{array}\right]=\left[\begin{array}{ccc}
1 & 1 & 1 \\
a^{2} & a & 1 \\
a & a^{2} & 1
\end{array}\right]\left[\begin{array}{l}
\dot{I}_{M k 1} \\
\dot{I}_{M k 2} \\
\dot{I}_{M k 0}
\end{array}\right],
$$




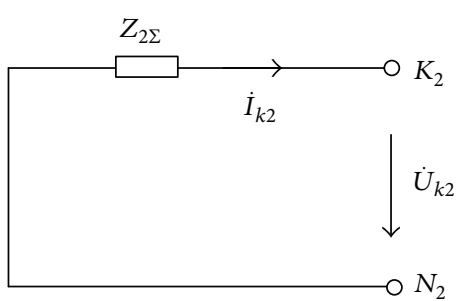

FIGURE 6: Simplified negative-sequence network.

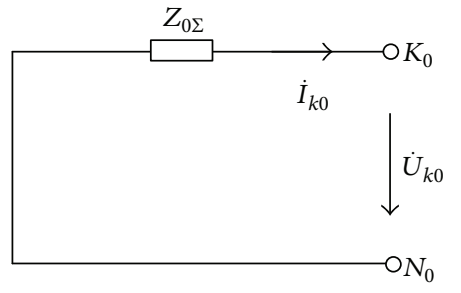

FIGURE 7: Simplified zero-sequence network.

where $a=e^{j 120^{\circ}}=-1 / 2+j(\sqrt{3} / 2)$ and $a^{2}=e^{j 240^{\circ}}=-1 / 2-$ $j(\sqrt{3} / 2)$.

Substituting (4) into (5), $\dot{I}_{M k a}, \dot{I}_{M k b}$, and $\dot{I}_{M k c}$ can be expressed by

$$
\begin{aligned}
\dot{I}_{M k a} & =\dot{I}_{M k 1}+\dot{I}_{M k 2}+\dot{I}_{M k 0} \\
& =C_{M 1} \dot{I}_{k 1}+C_{M 2} \dot{I}_{k 2}+C_{M 0} \dot{I}_{k 0} \\
& =C_{M 1}\left(\dot{I}_{k 1}+\dot{I}_{k 2}\right)+C_{M 0} \dot{I}_{k 0}, \\
\dot{I}_{M k b} & =a^{2} \dot{I}_{M k 1}+a \dot{I}_{M k 2}+\dot{I}_{M k 0} \\
& =a^{2} C_{M 1} \dot{I}_{k 1}+a C_{M 2} \dot{I}_{k 2}+C_{M 0} \dot{I}_{k 0} \\
& =C_{M 1}\left(a^{2} \dot{I}_{k 1}+a \dot{I}_{k 2}\right)+C_{M 0} \dot{I}_{k 0}, \\
\dot{I}_{M k c} & =a \dot{I}_{M k 1}+a^{2} \dot{I}_{M k 2}+\dot{I}_{M k 0} \\
& =C_{M 1}\left(a \dot{I}_{k 1}+a^{2} \dot{I}_{k 2}\right)+C_{M 0} \dot{I}_{k 0} .
\end{aligned}
$$

In the next subsection, aiming to simplify (6), we regard $\dot{I}_{k 1}$ as the known quantity to express $\dot{I}_{k 2}$ and $\dot{I}_{k 0}$ during different kinds of internal faults by means of method of symmetrical component as well as compound sequence network.

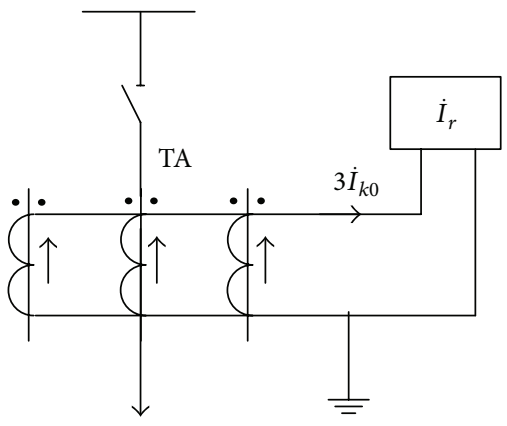

FigURE 8: Zero-sequence current measurement.

2.2. Derivation of 2 Ratios Characterizing Internal Short Circuit Faults. To meet the demand of the following mathematical derivation, we regard $\dot{I}_{M 0}$ as the zero-sequence current at the location of Protection 1 in Figure 1 and formulate the expression of $\dot{I}_{M 0}$ as follows:

$$
\dot{I}_{M 0}=3 C_{M 0} \dot{I}_{k 0}
$$

The reason to employ $3 C_{M 0} \dot{I}_{k 0}$ lies in the correspondence with the actual measurement of zero-sequence current by three-phase current transformers adopting star connection with neutral point, as is shown in Figure 8.

According to Figure 8, during the earth fault, the current flowing along the relay loop is

$$
\begin{aligned}
\dot{I}_{r}= & \dot{I}_{k}+\dot{I}_{k b}+\dot{I}_{k c} \\
= & \left(\dot{I}_{k 1}+\dot{I}_{k 2}+\dot{I}_{k 0}\right)+\left(a^{2} \dot{I}_{k 1}+a \dot{I}_{k 2}+\dot{I}_{k 0}\right) \\
& +\left(a \dot{I}_{k 1}+a^{2} \dot{I}_{k 2}+\dot{I}_{k 0}\right)=3 \dot{I}_{k 0},
\end{aligned}
$$

where $\dot{I}_{k b}$ and $\dot{I}_{k c}$ represent the $B$-phase and $C$-phase currents at point $K$.

Therefore, the actual electrical quantity applied to relay protection is three times the zero-sequence current. In digital protective device, three times the zero-sequence current can be obtained by superimposing three current phasors in the phase current formation loops. Equation (7) is for the convenience of derivation and application in the real protection device.

2.2.1. Modeling of Single-Phase Grounding Fault. In Figure 1, suppose an $A$-phase grounding fault occurs at point $K$. Select phase $A$ as the reference phase. According to symmetrical component method, the boundary condition at the fault point is

$$
\dot{I}_{k b}=\dot{I}_{k c}=0 .
$$

In order to simplify (6) under condition of single-phase grounding fault, we need to come up with the relationship 
among $\dot{I}_{k 1}, \dot{I}_{k 2}$, and $\dot{I}_{k 0}$. Similar to (5), $\dot{I}_{k a}, \dot{I}_{k b}$, and $\dot{I}_{k c}$ can be expressed by

$$
\begin{aligned}
{\left[\begin{array}{l}
\dot{I}_{k a} \\
\dot{I}_{k b} \\
\dot{I}_{k c}
\end{array}\right]=\left[\begin{array}{l}
\dot{I}_{k} \\
\dot{I}_{k b} \\
\dot{I}_{k c}
\end{array}\right]=\left[\begin{array}{lll}
1 & 1 & 1 \\
a^{2} & a & 1 \\
a & a^{2} & 1
\end{array}\right]\left[\begin{array}{l}
\dot{I}_{k 1} \\
\dot{I}_{k 2} \\
\dot{I}_{k 0}
\end{array}\right], } \\
{\left[\begin{array}{l}
\dot{I}_{k 1} \\
\dot{I}_{k 2} \\
\dot{I}_{k 0}
\end{array}\right]=\left[\begin{array}{ccc}
1 & 1 & 1 \\
a^{2} & a & 1 \\
a & a^{2} & 1
\end{array}\right]^{-1}\left[\begin{array}{l}
\dot{I}_{k a} \\
\dot{I}_{k b} \\
\dot{I}_{k c}
\end{array}\right] } \\
=\frac{1}{3}\left[\begin{array}{lll}
1 & a & a^{2} \\
1 & a^{2} & a \\
1 & 1 & 1
\end{array}\right]\left[\begin{array}{l}
\dot{I}_{k a} \\
\dot{I}_{k b} \\
\dot{I}_{k c}
\end{array}\right] .
\end{aligned}
$$

According to (9) and (11), we can get

$$
\dot{I}_{k 1}=\dot{I}_{k 2}=\dot{I}_{k 0}=\frac{1}{3} \dot{I}_{k a}
$$

Equation (6) can be simplified by substitution of (12) as

$$
\begin{aligned}
& \dot{I}_{M k a}=\left(2 C_{M 1}+C_{M 0}\right) \dot{I}_{k 1}, \\
& \dot{I}_{M k b}=\left(C_{M 0}-C_{M 1}\right) \dot{I}_{k 1}, \\
& \dot{I}_{M k c}=\left(C_{M 0}-C_{M 1}\right) \dot{I}_{k 1} .
\end{aligned}
$$

Equation (12) verifies the existence of zero-sequence current during a single-phase grounding fault. The zerosequence current at the location of Protection 1 can be expressed as (7).

Establish the models:

$$
\begin{aligned}
& \alpha=\frac{\max \left\{\left|\dot{I}_{M k a}\right|,\left|\dot{I}_{M k b}\right|,\left|\dot{I}_{M k c}\right|\right\}}{\min \left\{\left|\dot{I}_{M k a}\right|,\left|\dot{I}_{M k b}\right|,\left|\dot{I}_{M k c}\right|\right\}}, \\
& \beta=\frac{\left|\dot{I}_{M 0}\right|}{\max \left\{\left|\dot{I}_{M k a}\right|,\left|\dot{I}_{M k b}\right|,\left|\dot{I}_{M k c}\right|\right\}},
\end{aligned}
$$

where $\alpha$ and $\beta$ are two ratios to characterize internal asymmetrical short circuit fault.

Based on (13), we can come up with

$$
\begin{aligned}
& \alpha^{(1)}=\left|\frac{2 C_{M 1}+C_{M 0}}{C_{M 0}-C_{M 1}}\right|=\left|\frac{2+m}{m-1}\right|, \\
& \beta^{(1)}=\left|\frac{3 C_{M 0}}{2 C_{M 1}+C_{M 0}}\right|=\left|\frac{3 m}{2+m}\right|,
\end{aligned}
$$

where $\alpha^{(1)}$ and $\beta^{(1)}$ characterize single-phase grounding fault and $m=C_{M 0} / C_{M 1}$. When the fault location or the system operating mode varies, the value of $m$ will change. The curves of $\alpha^{(1)}$ versus $m$ and $\beta^{(1)}$ versus $m$ are shown as Figures 9 and 10 , respectively. No matter how big $m$ is, or no matter how the fault location or the system operating mode varies, either $\alpha^{(1)}$

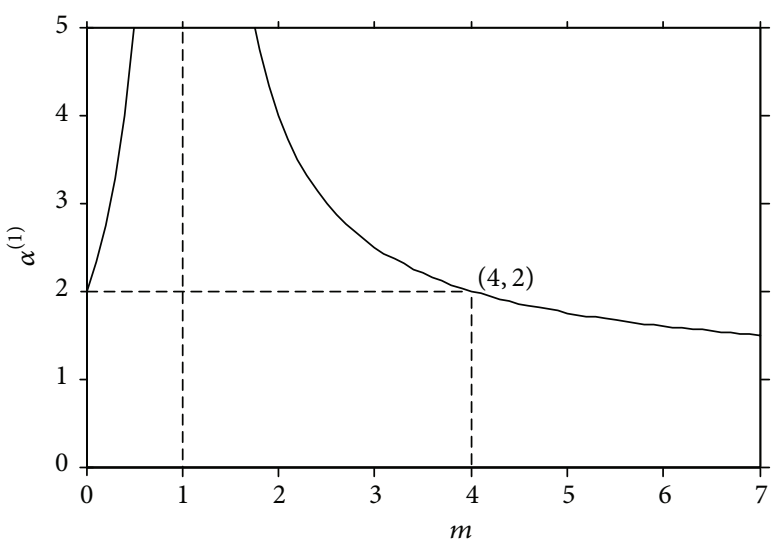

FIGURE 9: The curve of $\alpha^{(1)}$ versus $m$.

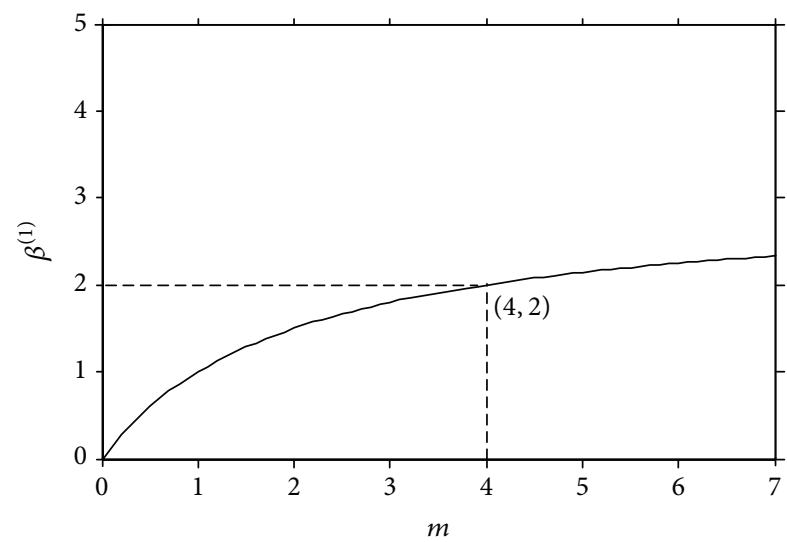

FIgURE 10: The curve of $\beta^{(1)}$ versus $m$.

or $\beta^{(1)}$ will keep larger than 2 , providing a theory basis for the settings of the identification criteria.

In the following modeling and derivation, aiming to provide a reliable theory foundation for the settings of the identification criteria, the similar process will be developed to solve the ranges of the ratios to characterize two-phase short circuit fault and two-phase grounding fault, which are calculated by the maximum/minimum fault component of 3 phase currents and zero-sequence current if it exists.

2.2.2. Modeling of Two-Phase Short Circuit Fault. Suppose that a short circuit between phase $B$ and phase $C$ occurs at point $K$ in Figure 1 . Select phase $A$ as the reference phase. Based on symmetrical component method, the boundary conditions at the short circuit point are

$$
\begin{aligned}
& \dot{I}_{k a}=0, \\
& \dot{I}_{k b}=-\dot{I}_{k c} .
\end{aligned}
$$




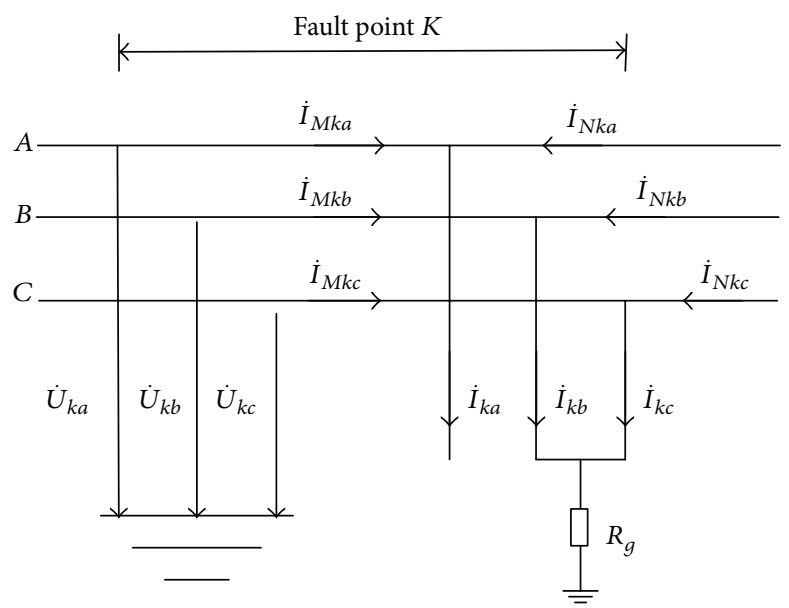

FIGURE 11: Power system diagram under condition of two-phase grounding fault.

Substitute (16) into (11):

$$
\begin{aligned}
& \dot{I}_{k 1}=\frac{1}{3}\left(\dot{I}_{k a}+a \dot{I}_{k b}+a^{2} \dot{I}_{k c}\right)=j \frac{1}{\sqrt{3}} \dot{I}_{k b}, \\
& \dot{I}_{k 2}=\frac{1}{3}\left(\dot{I}_{k a}+a^{2} \dot{I}_{k b}+a \dot{I}_{k c}\right)=-j \frac{1}{\sqrt{3}} \dot{I}_{k b}, \\
& \dot{I}_{k 0}=\frac{1}{3}\left(\dot{I}_{k a}+\dot{I}_{k b}+\dot{I}_{k c}\right)=0 .
\end{aligned}
$$

Therefore

$$
\begin{aligned}
& \dot{I}_{k 1}=-\dot{I}_{k 2}, \\
& \dot{I}_{k 0}=0 .
\end{aligned}
$$

Equation (6) can be simplified by substitution of (18) as

$$
\begin{aligned}
& \dot{I}_{M k a}=0 \\
& \dot{I}_{M k b}=C_{M 1}\left(a^{2}-a\right) \dot{I}_{k 1}=-j \sqrt{3} C_{M 1} \dot{I}_{k 1}, \\
& \dot{I}_{M k c}=C_{M 1}\left(a-a^{2}\right) \dot{I}_{k 1}=j \sqrt{3} C_{M 1} \dot{I}_{k 1} .
\end{aligned}
$$

Based on (18), during a short circuit between phase $B$ and phase $C, \dot{I}_{M 0}=3 C_{M 0} \dot{I}_{k 0}=0$.

Define

$$
\begin{aligned}
& \alpha^{(2)}=\frac{\max \left\{\left|\dot{I}_{M k a}\right|,\left|\dot{I}_{M k b}\right|,\left|\dot{I}_{M k c}\right|\right\}}{\min \left\{\left|\dot{I}_{M k a}\right|,\left|\dot{I}_{M k b}\right|,\left|\dot{I}_{M k c}\right|\right\}}=\infty, \\
& \beta^{(2)}=\frac{\left|\dot{I}_{M 0}\right|}{\max \left\{\left|\dot{I}_{M k a}\right|,\left|\dot{I}_{M k b}\right|,\left|\dot{I}_{M k c}\right|\right\}}=0,
\end{aligned}
$$

where $\alpha^{(2)}$ and $\beta^{(2)}$ characterize two-phase short circuit fault. Consequently, during two-phase short circuit fault, $\alpha^{(2)}$ tends to be positive infinite because the denominator $\min \left\{\left|\dot{I}_{M k a}\right|,\left|\dot{I}_{M k b}\right|,\left|\dot{I}_{M k c}\right|\right\}$ is 0 , and $\beta^{(2)}$ is equal to 0 because $\dot{I}_{M 0}=0$, wherever the fault position is or whatever the system operating mode is.
2.2.3. Modeling of Two-Phase Grounding Fault. As is shown in Figure 11, suppose that a short circuit between phase $B$ and phase $C$ occurs at point $K$ in Figure 1 , and point $K$ is connected to the ground via the transition resistance $R_{g}$.

Select phase $A$ as the reference phase. Based on symmetrical component method, the boundary conditions at the fault point are

$$
\begin{aligned}
& \dot{I}_{k a}=\dot{I}_{k 1}+\dot{I}_{k 2}+\dot{I}_{k 0}=0, \\
& \dot{U}_{k b}=\dot{U}_{k c} \\
& \dot{U}_{k b}=\left(\dot{I}_{k b}+\dot{I}_{k c}\right) R_{g}
\end{aligned}
$$

where $\dot{U}_{k b}$ and $\dot{U}_{k c}$ represent the $B$-phase and $C$-phase voltages to ground at point $K$.

Similar to (5), $\dot{U}_{k b}$ and $\dot{U}_{k c}$ can be expressed as

$$
\left[\begin{array}{c}
\dot{U}_{k b} \\
\dot{U}_{k c}
\end{array}\right]=\left[\begin{array}{lll}
a^{2} & a & 1 \\
a & a^{2} & 1
\end{array}\right]\left[\begin{array}{c}
\dot{U}_{k 1} \\
\dot{U}_{k 2} \\
\dot{U}_{k 0}
\end{array}\right],
$$

where $\dot{U}_{k 1}, \dot{U}_{k 2}$, and $\dot{U}_{k 3}$ represent the positive-sequence, negative-sequence, and zero-sequence components of $A$ phase voltage to ground at point $K$, respectively.

Solve (22) and (24) simultaneously:

$$
a^{2} \dot{U}_{k 1}+a \dot{U}_{k 2}+\dot{U}_{k 0}=a \dot{U}_{k 1}+a^{2} \dot{U}_{k 2}+\dot{U}_{k 0} .
$$

That is,

$$
\begin{aligned}
\dot{U}_{k 1} & =\dot{U}_{k 2}, \\
\dot{U}_{k b} & =a^{2} \dot{U}_{k 1}+a \dot{U}_{k 2}+\dot{U}_{k 0}=\left(a^{2}+a\right) \dot{U}_{k 1}+\dot{U}_{k 0} \\
& =-\dot{U}_{k 1}+\dot{U}_{k 0} .
\end{aligned}
$$




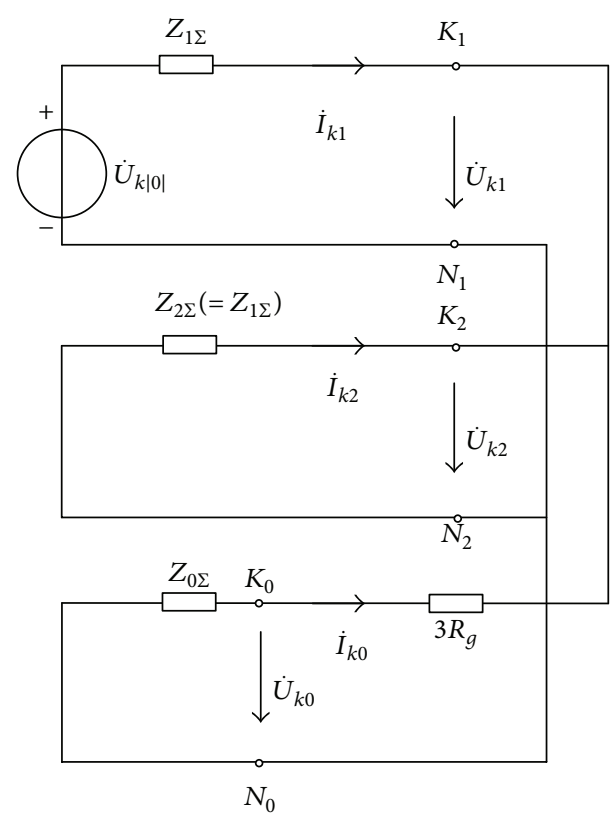

Figure 12: Compound sequence network under condition of twophase grounding fault.

On the other hand, based on (23) and (24),

$$
\begin{aligned}
\dot{U}_{k b} & =\left(\dot{I}_{k b}+\dot{I}_{k c}\right) Z_{g} \\
& =\left(a^{2} \dot{I}_{k 1}+a \dot{I}_{k 2}+\dot{I}_{k 0}+a \dot{I}_{k 1}+a^{2} \dot{I}_{k 2}+\dot{I}_{k 0}\right) R_{g} \\
& =\left(\dot{I}_{k a}+3 \dot{I}_{k 0}\right) R_{g}=3 \dot{I}_{k 0} R_{g} .
\end{aligned}
$$

Therefore

$$
-\dot{U}_{k 1}+\dot{U}_{k 0}=3 \dot{I}_{k 0} R_{g} .
$$

To solve $\dot{I}_{k 1}, \dot{I}_{k 2}$, and $\dot{I}_{k 0}$, we apply the method of compound sequence network formed by joining 3 simplified sequence networks shown in Figures 3, 6, and 7 in terms of boundary conditions expressed by sequence components at fault point. Based on (23), (27), and (30), we should connect 3 simplified sequence networks in parallel after having the zero-sequence network in series with $3 R_{g}$ to obtain the compound sequence network, which is shown in Figure 12.

From Figure 12, we can obtain

$$
\begin{aligned}
& \dot{I}_{k 1}=\frac{\dot{U}_{k|0|}}{Z_{1 \Sigma}+Z_{1 \Sigma}\left(Z_{0 \Sigma}+3 R_{g}\right) /\left(Z_{1 \Sigma}+Z_{0 \Sigma}+3 R_{g}\right)}, \\
& \dot{I}_{k 2}=-\frac{Z_{0 \Sigma}+3 R_{g}}{Z_{1 \Sigma}+Z_{0 \Sigma}+3 R_{g}} \dot{I}_{k 1}, \\
& \dot{I}_{k 0}=-\frac{Z_{1 \Sigma}}{Z_{1 \Sigma}+Z_{0 \Sigma}+3 R_{g}} \dot{I}_{k 1} .
\end{aligned}
$$

Substitute (29)-(31) into (6):

$$
\begin{aligned}
& \dot{I}_{M k a}=C_{M 1}\left(\dot{I}_{k 1}+\dot{I}_{k 2}+\dot{I}_{k 0}\right)+\left(C_{M 0}-C_{M 1}\right) \dot{I}_{k 0} \\
& =\left(C_{M 1}-C_{M 0}\right) \frac{Z_{1 \Sigma}}{Z_{1 \Sigma}+Z_{0 \Sigma}+3 R_{g}} \dot{I}_{k 1}=\left(C_{M 1}\right. \\
& \left.-C_{M 0}\right) \frac{\dot{U}_{k|0|}}{Z_{1 \Sigma}+2\left(Z_{0 \Sigma}+3 R_{g}\right)} \text {, } \\
& \dot{I}_{M k b}=C_{M 1} \dot{I}_{k 1}\left(a^{2}-a \frac{Z_{0 \Sigma}+3 R_{g}}{Z_{1 \Sigma}+Z_{0 \Sigma}+3 R_{g}}\right)-C_{M 0} \\
& \cdot \frac{Z_{1 \Sigma}}{Z_{1 \Sigma}+Z_{0 \Sigma}+3 R_{g}} \dot{I}_{k 1} \\
& =\frac{\dot{I}_{k 1}}{Z_{1 \Sigma}+Z_{0 \Sigma}+3 R_{g}}\left[C_{M 1} a^{2} Z_{1 \Sigma}\right. \\
& \left.+C_{M 1}\left(a^{2}-a\right)\left(Z_{0 \Sigma}+3 R_{g}\right)-C_{M 0} Z_{1 \Sigma}\right] \\
& =-\frac{\dot{U}_{k|0|}}{Z_{1 \Sigma}+2\left(Z_{0 \Sigma}+3 R_{g}\right)}\left(\frac{C_{M 1}}{2}+C_{M 0}\right)-j \frac{\sqrt{3}}{2} \\
& \cdot \frac{\dot{U}_{k|0|} C_{M 1}}{Z_{1 \Sigma}}=X+j Y \text {, } \\
& \dot{I}_{M k c}=C_{M 1} \dot{I}_{k 1}\left(a-a^{2} \frac{Z_{0 \Sigma}+3 R_{g}}{Z_{1 \Sigma}+Z_{0 \Sigma}+3 R_{g}}\right)-C_{M 0} \\
& \cdot \frac{Z_{1 \Sigma}}{Z_{1 \Sigma}+Z_{0 \Sigma}+3 R_{g}} \dot{I}_{k 1} \\
& =\frac{\dot{I}_{k 1}}{Z_{1 \Sigma}+Z_{0 \Sigma}+3 R_{g}}\left[C_{M 1} a Z_{1 \Sigma}\right. \\
& \left.+C_{M 1}\left(a-a^{2}\right)\left(Z_{0 \Sigma}+3 R_{g}\right)-C_{M 0} Z_{1 \Sigma}\right] \\
& =-\frac{\dot{U}_{k|0|}}{Z_{1 \Sigma}+2\left(Z_{0 \Sigma}+3 R_{g}\right)}\left(\frac{C_{M 1}}{2}+C_{M 0}\right)+j \frac{\sqrt{3}}{2} \\
& \text {. } \frac{\dot{U}_{k|0|} C_{M 1}}{Z_{1 \Sigma}}=X-j Y \text {, }
\end{aligned}
$$

where $X=-\left(\dot{U}_{k|0|} /\left(Z_{1 \Sigma}+2\left(Z_{0 \Sigma}+3 R_{g}\right)\right)\right)\left(C_{M 1} / 2+C_{M 0}\right)$ and $Y=-(\sqrt{3} / 2)\left(\dot{U}_{k|0|} C_{M 1} / Z_{1 \Sigma}\right)$. So $\left|\dot{I}_{M k b}\right|=\sqrt{X^{2}+Y^{2}}=\left|\dot{I}_{M k c}\right|$.

Consider

$$
\begin{aligned}
& \left|\frac{\dot{I}_{M k b}}{\dot{I}_{M k a}}\right|=\left|\frac{\dot{I}_{M k c}}{\dot{I}_{M k a}}\right| \\
& =\mid j \frac{\sqrt{3}}{2} \frac{C_{M 1}}{C_{M 0}-C_{M 1}}\left(1+2 \times \frac{Z_{0 \Sigma}+3 R_{g}}{Z_{1 \Sigma}}\right) \\
& +\frac{1}{2} \frac{C_{M 1}+2 C_{M 0}}{C_{M 0}-C_{M 1}}|=| \frac{1}{2} \frac{1+2 m}{m-1} \\
& +j \frac{\sqrt{3}}{2} \frac{1}{m-1}(1+2 n) \\
& =\frac{\sqrt{(2 m+1)^{2}+3(1+2 n)^{2}}}{2|m-1|},
\end{aligned}
$$


where $n=\left(Z_{0 \Sigma}+3 R_{q}\right) / Z_{1 \Sigma}$. Since $\sqrt{(2 m+1)^{2}+3(1+2 n)^{2}}>$ $\sqrt{4 m^{2}+4 m+4}, 2|m-1|=\sqrt{4 m^{2}+4-8 m}$, so $\left|\dot{I}_{M k b} / \dot{I}_{M k a}\right|=$ $\left|\dot{I}_{M k c}\right| \dot{I}_{M k a} \mid>1$.

Therefore

$$
\begin{aligned}
\alpha^{(1,1)} & =\frac{\max \left\{\left|\dot{I}_{M k a}\right|,\left|\dot{I}_{M k b}\right|,\left|\dot{I}_{M k c}\right|\right\}}{\min \left\{\left|\dot{I}_{M k a}\right|,\left|\dot{I}_{M k b}\right|,\left|\dot{I}_{M k c}\right|\right\}}=\left|\frac{\dot{I}_{M k b}}{\dot{I}_{M k a}}\right| \\
& =\left|\frac{\dot{I}_{M k c}}{\dot{I}_{M k a}}\right|=\frac{\sqrt{(2 m+1)^{2}+3(1+2 n)^{2}}}{2|m-1|},
\end{aligned}
$$

where $\alpha^{(1,1)}$ characterizes two-phase short circuit fault. The values of $m$ and $n$ will change with the different position of point $K$. Figure 13 shows the curves of $\alpha^{(1,1)}$ versus $m$ and $n$. With regard to a fixed $n$, when $0<m<1, \alpha^{(1,1)}$ increases monotonically; when $m>1$, it decreases monotonically. With regard to a fixed $m$, the larger $n$ is, the larger the value of $\alpha^{(1,1)}$ will be. Taking $n=0.5,1,1.5$ as representatives, the curves of $\alpha^{(1,1)}$ versus $m$ are shown in Figure 14. In the range of $m<3.4, \alpha^{(1,1)}$ will keep larger than 1.778:

$$
\begin{aligned}
\dot{I}_{M 0} & =3 C_{M 0} \dot{I}_{k 0}=-\frac{3 C_{M 0} \dot{U}_{k|0|}}{Z_{1 \Sigma}+2\left(Z_{0 \Sigma}+3 Z_{g}\right)}, \\
\beta^{(1,1)} & =\frac{\left|\dot{I}_{M 0}\right|}{\max \left\{\left|\dot{I}_{M k a}\right|,\left|\dot{I}_{M k b}\right|,\left|\dot{I}_{M k c}\right|\right\}}=\frac{\left|\dot{I}_{M 0}\right|}{\left|\dot{I}_{M k b}\right|} \\
& =\frac{\left|\dot{I}_{M 0}\right|}{\left|\dot{I}_{M k c}\right|}=\left|\frac{6 m}{(1+2 m)+j \sqrt{3}(1+2 n)}\right| \\
& =\frac{6 m}{\sqrt{(1+2 m)^{2}+3(1+2 n)^{2}}},
\end{aligned}
$$

where $\beta^{(1,1)}$ also characterizes two-phase short circuit fault. Figure 15 shows the curves of $\beta^{(1,1)}$ versus $m$ and $n$. Fixing $n$, we can see that the larger $m$ is, the larger the value of $\beta^{(1,1)}$ will be. Fixing $m, \beta^{(1,1)}$ decreases monotonically with the increment of $n$. Taking $n=0.5,1,1.5$ as well, the curves of $\beta^{(1,1)}$ versus $m$ are shown as Figure 16. In the range of $m>1$, $\beta^{(1,1)}$ will keep no smaller than 0.795 .

A comprehensive synthesis of values of $\alpha^{(1,1)}$ and $\beta^{(1,1)}$ belonging to different ranges of $m$ or $n$ is listed in Table 1. For most of the two-phase grounding faults, as well as all of singlephase grounding faults and two-phase short circuit faults, at least one of $\alpha^{(1,1)}>1.778$ and $\beta^{(1,1)}>0.795$ will be satisfied. Under condition that $n<0.5$ and $m \ll 1$, neither of them may not be satisfied; then we will use the third ratio proposed in Section 3 to characterize the two-phase grounding fault.

Based on current distribution factor theory and asymmetric short circuit fault modeling, the derivation on ranges of $\alpha$ and $\beta$ under all types of asymmetric short circuit

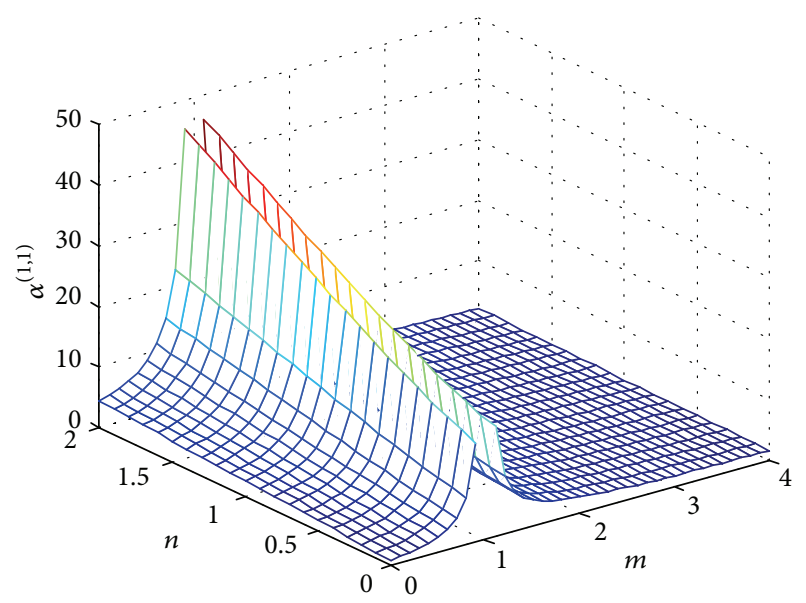

FIGURE 13: The curves of $\alpha^{(1,1)}$ versus $m$ and $n$.

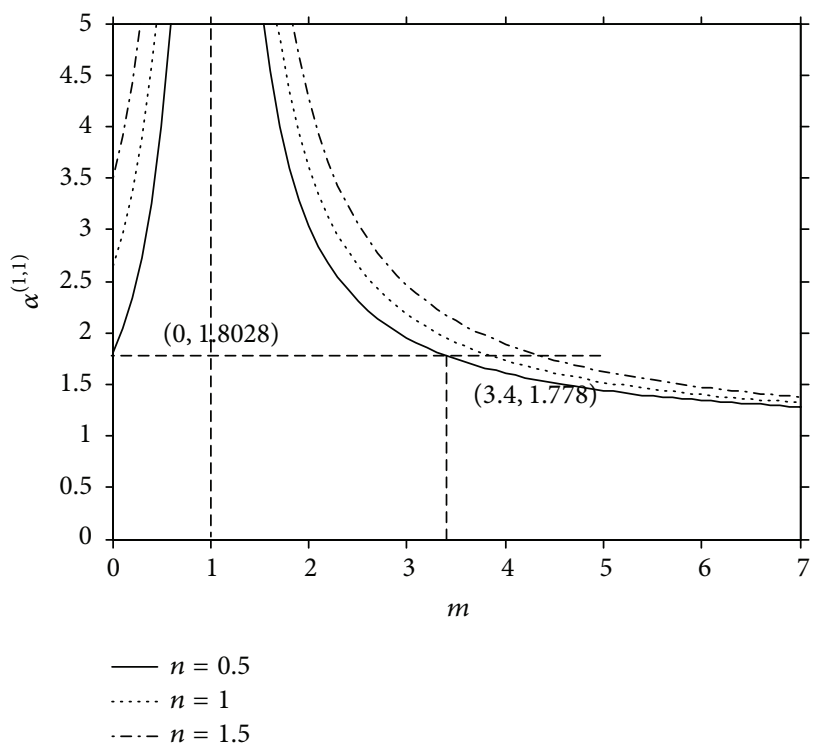

FIGURE 14: The curves of $\alpha^{(1,1)}$ versus $m$.

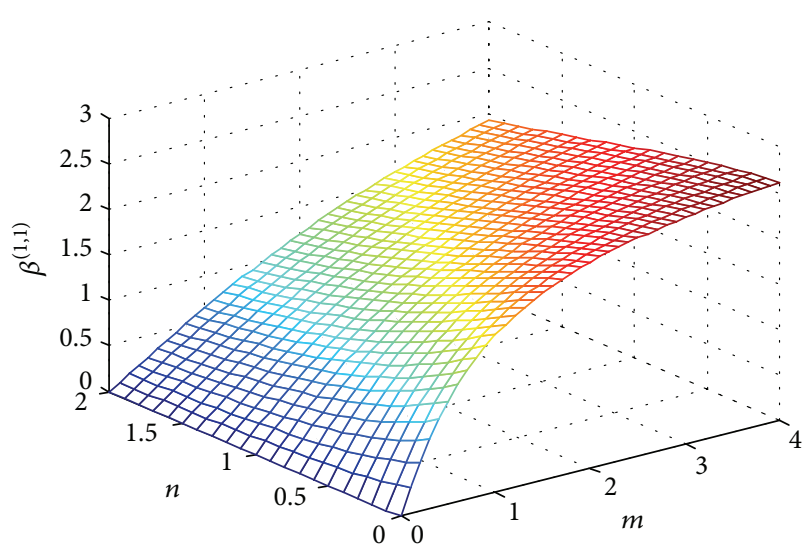

Figure 15: The curves of $\beta^{(1,1)}$ versus $m$ and $n$. 


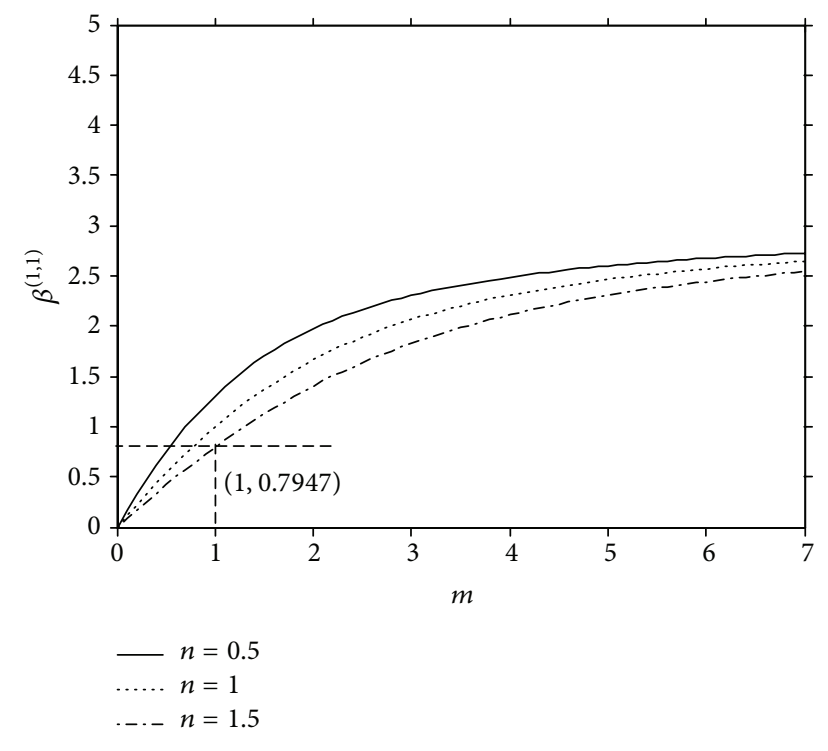

FIgURE 16: The curves of $\beta^{(1,1)}$ versus $m$.

TABLE 1: Ranges of $\alpha^{(1,1)}$ and $\beta^{(1,1)}$.

\begin{tabular}{lccc}
\hline & $m \leq 1$ & $1<m \leq 3.4$ & $m>3.4$ \\
\hline$n<0.5$ & - & $\alpha^{(1,1)}>1.778$ & $\beta^{(1,1)}>0.795$ \\
$0.5<n<1.5$ & $\alpha^{(1,1)}>1.778$ & $\beta^{(1,1)}>0.795$ & \\
$n>1.5$ & & & \\
\hline
\end{tabular}

faults has provided a reliable theory basis for the current identification criteria, which are established as follows:

$$
\begin{aligned}
& \alpha>k_{1}, \\
& \beta>k_{2},
\end{aligned}
$$

where $\alpha=\max \left\{\left|\dot{I}_{M k a}\right|,\left|\dot{I}_{M k b}\right|,\left|\dot{I}_{M k c}\right|\right\} / \min \left\{\left|\dot{I}_{M k a}\right|,\left|\dot{I}_{M k b}\right|\right.$, $\left.\left|\dot{I}_{M k c}\right|\right\}$ and $\beta=\left|\dot{I}_{M 0}\right| / \max \left\{\left|\dot{I}_{M k a}\right|,\left|\dot{I}_{M k b}\right|,\left|\dot{I}_{M k c}\right|\right\}$, in which $\dot{I}_{M k a}, \dot{I}_{M k b}$, and $\dot{I}_{M k c}$ are the actual fault components of 3 phase currents obtained by

$$
\dot{I}_{M k \varphi}=\dot{I}_{M \varphi}-\dot{I}_{M \varphi|0|}, \quad \varphi=a, b, c,
$$

where $\dot{I}_{M \varphi}$ and $\dot{I}_{M \varphi|0|}$ represent the postfault and prefault phase currents measured by WAMS at the location of protection, respectively; $\dot{I}_{M 0}$ represents the WAMS-measured zerosequence current at the location of protection; $k_{1}$ and $k_{2}$ can not only be suggested as 1.778 and 0.795 before relays are put in use, but also be determined in terms of the onthe-spot conditions. The 2 criteria (36) will always work whatever the system operating mode is and wherever the fault is located. Either of the two current criteria is satisfied can the asymmetric fault be identified.

\section{Mathematical Derivation of Criteria Based on Fault Voltage Model}

When a two-phase short circuit fault occurs, the voltage between faulted phases at fault point called arc voltage is lower than 5 percent of the rated voltage, and it has nothing to do with the location of fault point. This is called the Characteristic of Arc Voltage [22]. Since three-phase short circuit is frequently evolved from two-phase short circuit, the arc voltage on the interphase transition resistance (mainly including arc resistance) between any two faulted phases during a three-phase short circuit fault also owns the same characteristic. Yet if the overload caused by transferred power flow occurs on a transmission line, the interphase voltage is relatively much higher. In this section, taking advantage of the characteristic of arc voltage, the third characteristic ratio computed by the WAMS-measured interphase voltage and impedance angle at the location of backup protection is derived mathematically via fault voltage model. The voltage criterion will be set up to distinguish between phase-to-phase fault and nonfault overload to perfect the novel algorithm concluded in Section 4.

Suppose that $E_{M i j}$ and $E_{N i j}$ represent the $M$-side and $N$-side rated voltages. Analysis below is on the basis of the assumptions that $E_{M i j}=E_{N i j}$ and $\arg \left(Z_{M}\right)=\arg \left(Z_{N}\right)=$ $\arg \left(Z_{M M^{\prime}}\right)=\arg \left(Z_{M^{\prime} N}\right)$. Even though they are not strictly true, there is little impact on the derived conclusion.

Suppose that a symmetric fault (three-phase short circuit fault) occurs at point $K$. At Protection 2, the interphase voltage represented by $\dot{U}_{M^{\prime} i j}(i j=a b, b c, c a)$ is

$$
\dot{U}_{M^{\prime} i j}=\dot{I}_{M^{\prime} i j} Z_{M^{\prime} K}+\dot{U}_{\text {arc }}
$$

where $\dot{I}_{M^{\prime} i j}$ represents the phase-to-phase differential current measured at Protection 2 and $\dot{U}_{\text {arc }}$ is the arc voltage at the fault point. Figure 17 shows the relationship among phasors on Line 2 under condition of three-phase short circuit fault. $\varphi_{L 2}$ is the positive-sequence impedance angle of Line 2. $O A$ represents the distance from point $O$ to the extension of $\dot{I}_{M^{\prime} i j} Z_{M^{\prime} K}$.

The length of $O A$ can be calculated by

$$
\overline{O A}=\left|U_{M^{\prime} i j} \cos \varphi_{M^{\prime} i j}\right|,
$$

where $\varphi_{M^{\prime} i j}=\arg \left(\dot{U}_{M^{\prime} i j} / \dot{I}_{M^{\prime} i j}\right)+90^{\circ}-\varphi_{L 2}$. Based on geometrical principle, $O A$ is shorter than $\left|\dot{U}_{\text {arc }}\right|$, so $\overline{O A}$ is smaller than $5 \% E_{M}$.

As zone 3 distance relays of Protection 1 are the backup of Protection 2, based on Kirchhoff voltage law, the interphase voltage at Protection 1 which is represented by $\dot{U}_{M i j}$ is

$$
\dot{U}_{M i j}=\dot{I}_{M i j} Z_{M M^{\prime}}+\dot{I}_{M^{\prime} i j} Z_{M^{\prime} K}+\dot{U}_{\mathrm{arc}}
$$

where $\dot{I}_{M i j}$ represents the phase-to-phase differential current measured at Protection 1. Figure 18 shows the relationship among phasors in (40). $\varphi_{L 1}$ is the positive-sequence impedance angle of Line 1; $O B$ represents the distance from point $O$ to the extension of $\dot{I}_{M i j} Z_{M M^{\prime}}$.

Learning from (39), the length of $O B$ can be calculated by

$$
\overline{O B}=\left|U_{M i j} \cos \varphi_{M i j}\right|,
$$

where $\varphi_{M i j}=\arg \left(\dot{U}_{M i j} / \dot{I}_{M i j}\right)+90^{\circ}-\varphi_{L 1}$. 


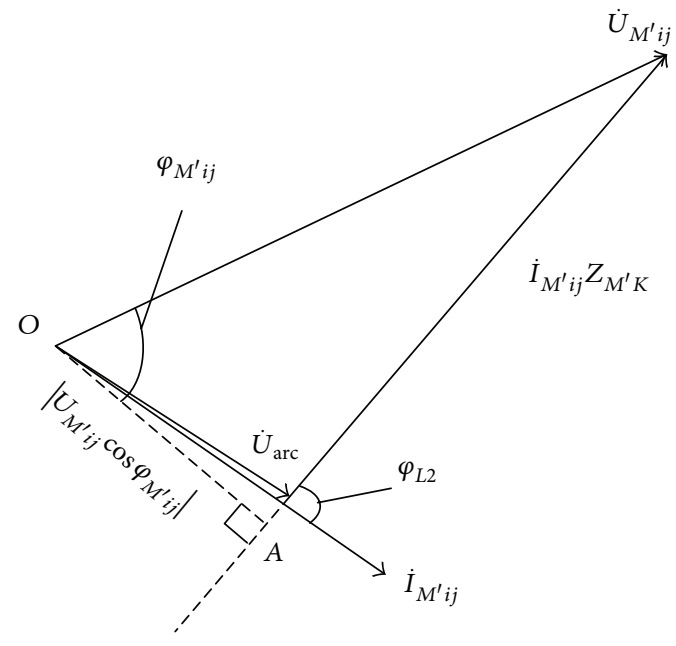

FIgURE 17: Phasor diagram at Protection 2 under condition of threephase short circuit fault.

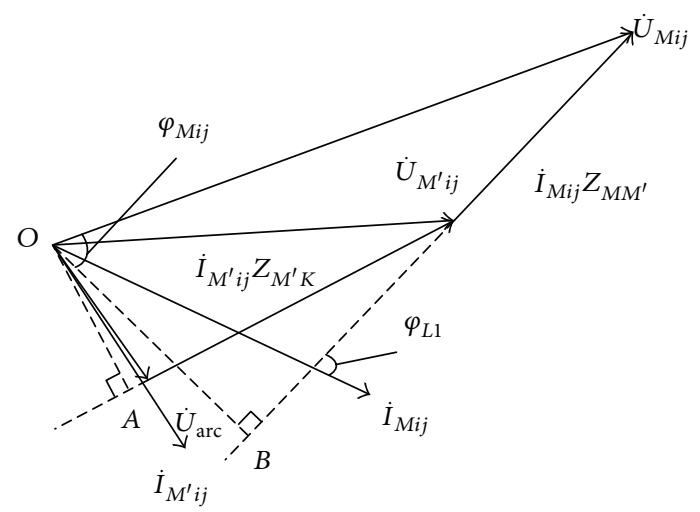

FIGURE 18: Phasor diagram at Protection 1 under condition of threephase short circuit fault.

Obviously, $U_{M i j}<E_{M i j}$ and the value of $\overline{O B}$ partially depends on $\varphi_{M i j}$, or $\arg \left(\dot{U}_{M i j} / \dot{I}_{M i j}\right)$, which is correlative to the impedance property of load connected to Bus $M^{\prime}$ that the stronger the resistiveness of the load impedance is, the smaller $\arg \left(\dot{U}_{M i j} / \dot{I}_{M i j}\right)$ will be and the larger the value of $\overline{O B}$ will be. In order to solve $\min \arg \left(\dot{U}_{M i j} / \dot{I}_{M i j}\right)$, the limit thought in mathematics is applied. Assume that the equivalent impedance of load connected to Bus $M^{\prime}$ is purely resistive and is represented by $R_{\text {load }}$. Regard $\dot{I}_{\text {load } i j}$ as the phase-to-phase differential current of the load branch. Assume that the line impedance is purely inductive; in other words, $Z_{M M^{\prime}}=j X_{M M^{\prime}}$ and $Z_{M^{\prime} K}=j X_{M^{\prime} K}$. Regard $\dot{I}_{M^{\prime} K i j}$ as the phase-to-phase differential current of Line 2. Ignore the arc resistance. Taking the lines connected to Bus $M^{\prime}$ into account, the branch coefficient $K_{b}$ is defined as

$$
K_{b}=\frac{\dot{I}_{s i j}}{\dot{I}_{M i j}},
$$

where $\dot{I}_{s i j}=\dot{I}_{M^{\prime} K i j}+\dot{I}_{\text {load } i j}$.
Based on Kirchhoff voltage law,

$$
\begin{aligned}
\dot{U}_{M i j} & =\dot{I}_{M i j} j X_{M M^{\prime}}+\frac{\dot{I}_{s i j}}{\left(1 / j X_{M^{\prime} K}+1 / R_{\text {load }}\right)} \\
& =\dot{I}_{M i j}\left[j X_{M M^{\prime}}+\frac{K_{b}}{\left(1 / j X_{M^{\prime} K}+1 / R_{\text {load }}\right)}\right] .
\end{aligned}
$$

Thus, $\arg \left(\dot{U}_{M i j} / \dot{I}_{M i j}\right)$ can be solved by

$$
\begin{aligned}
& \arg \left(\frac{\dot{U}_{M i j}}{\dot{I}_{M i j}}\right)=\arg \left[j X_{M M^{\prime}}+\frac{K_{b}}{\left(1 / j X_{M^{\prime} K}+1 / R_{\text {load }}\right)}\right] \\
& \quad=\arg \left[j \left(K_{b} X_{M^{\prime} K} R_{\text {load }}^{2}+X_{M M^{\prime}} R_{\text {load }}^{2}\right.\right. \\
& \left.\left.\quad+X_{M M^{\prime}} X_{M^{\prime} K}^{2}\right)+K_{b} R_{\text {load }} X_{M^{\prime} K}^{2}\right], \\
& \tan \left[\arg \left(\frac{\dot{U}_{M i j}}{\dot{I}_{M i j}}\right)\right]=\frac{R_{\text {load }}\left(X_{M M^{\prime}}+K_{b} X_{M^{\prime} K}\right)}{K_{b} X_{M^{\prime} K}^{2}} \\
& \quad+\frac{X_{M M^{\prime}}}{K_{b} R_{\text {load }}}
\end{aligned}
$$

For (45), according to the formation of mathematical basic inequality expressed as $e+f \geq 2 \sqrt{e f}, e, f \geq 0$, only when $R_{\text {load }}\left(X_{M M^{\prime}}+K_{b} X_{M^{\prime} K}\right) /\left(K_{b} X_{M^{\prime} K}^{2}\right)=X_{M M^{\prime}} /\left(K_{b} R_{\text {load }}\right)$ can $\tan \left[\arg \left(\dot{U}_{M i j} / \dot{I}_{M i j}\right)\right]$ reach the minimum:

$$
\begin{aligned}
& \min \tan \left[\arg \left(\frac{\dot{U}_{M i j}}{\dot{I}_{M i j}}\right)\right] \\
& =\frac{2 \sqrt{X_{M M^{\prime}}\left(X_{M M^{\prime}}+K_{b} X_{M^{\prime} K}\right)}}{K_{b} X_{M^{\prime} K}} .
\end{aligned}
$$

Figure 19 shows the curves of $\min \tan \left[\arg \left(\dot{U}_{M i j} / \dot{I}_{M i j}\right)\right]$ versus $K_{b}$ under conditions of $X_{M M^{\prime}} / X_{M^{\prime} K}=0.5,1,1.5,2$.

As illustrated in Figure 19, the smaller $X_{M M^{\prime}} / X_{M^{\prime} K}$ is and the larger $K_{b}$ is, the smaller $\min \tan \left[\arg \left(\dot{U}_{M i j} / \dot{I}_{M i j}\right)\right]$ will be. In the lower right area, $\min \tan \left[\arg \left(\dot{U}_{M i j} / \dot{I}_{M i j}\right)\right]=$ 0.88 with $X_{M M^{\prime}} / X_{M^{\prime} K}=0.5$ and $K_{b}=3$. Because the monotonicity of $\min \tan \left[\arg \left(\dot{U}_{M i j} \dot{I}_{M i j}\right)\right]$ is consistent with that of $\arg \left(\dot{U}_{M i j} / \dot{I}_{M i j}\right), \arg \left(\dot{U}_{M i j} / \dot{I}_{M i j}\right)$ also reaches the minimum of about $41.4^{\circ}$. Considering the influence of the grounding resistance $[23,24]$, the $\min \arg \left(\dot{U}_{M i j} / \dot{I}_{M i j}\right)$ is suggested to be $48^{\circ}$, or it can be adjusted in terms of real transmission line and load conditions. Based on the analysis above, $\cos \arg \left(\dot{U}_{M i j} / \dot{I}_{M i j}\right)$ will keep smaller than about 0.669 during a three-phase short circuit fault. Based on the per unit (p.u.) system ( $E_{M i j}$ is the reference value), we can obtain

$$
\left|U_{M i j} \cos \varphi_{M i j}\right|<0.669 \text { p.u. }
$$

Inequality (47) is also applied to the circumstances of both two-phase grounding fault and two-phase short circuit fault because derivations under these circumstances can be carried out in the same way as under condition of three-phase 


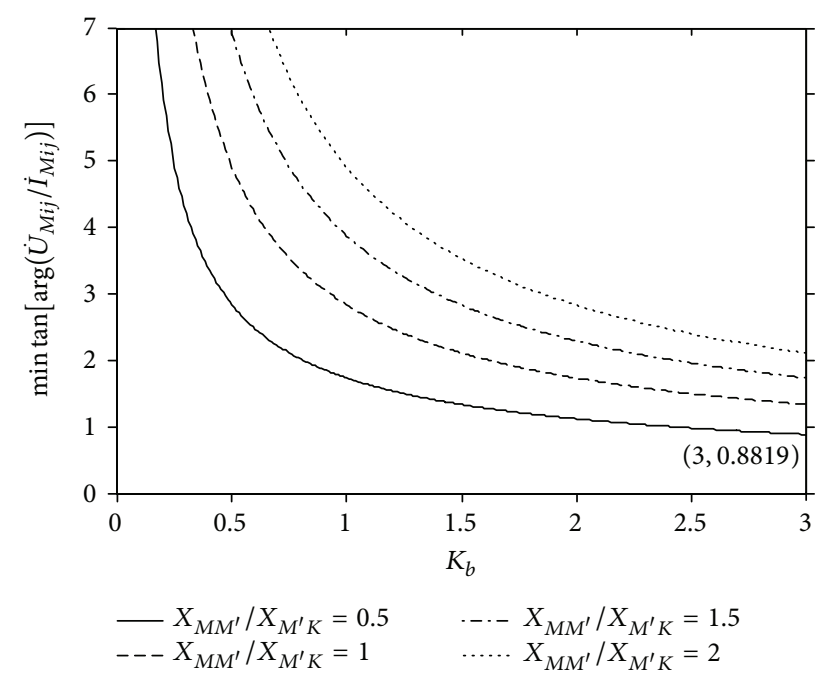

Figure 19: The curves of $\min \tan \left[\arg \left(\dot{U}_{M i j} / \dot{I}_{M i j}\right)\right]$ versus $K_{b}$.

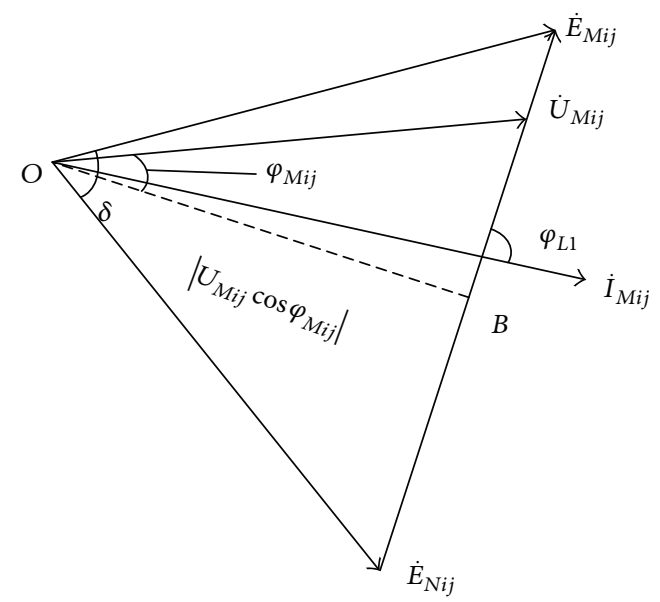

Figure 20: Phasor diagram under condition of overload caused by flow transferring.

short circuit fault. What is different from the process above is that three-phase voltages at the relay point are asymmetric, so (47) should be modified as

$$
\min \left|U_{M i j} \cos \varphi_{M i j}\right|<0.669 \text { p.u. }
$$

In the novel algorithm, $\overline{O B}$ is constructed to reflect the severity of overload. When a phase-to-phase fault occurs, $\overline{O B}$ will keep smaller than 66.9 percent of the rated voltage, while under condition of overload caused by flow transferring, as is shown in Figure 20, $\overline{O B}$ will keep larger than 66.9 percent of the rated.

Based on Figure 20, the expression in geometry is

$$
\begin{aligned}
\left|E_{M i j} \cos \frac{\delta}{2}\right| & =\overline{O B}=\left|U_{M i j} \cos \varphi_{M i j}\right|, \\
\left|\cos \frac{\delta}{2}\right| & =\left|\frac{U_{M i j}}{E_{M i j}} \cos \varphi_{M i j}\right| .
\end{aligned}
$$

When transmission lines are unloaded, $\delta=0^{\circ}$, so $\left|U_{M i j} \cos \varphi_{M i j}\right|=1$ p.u., while when power system is on the boundary of static steady, $\delta=90^{\circ}$, so $\left|U_{M i j} \cos \varphi_{M i j}\right|=$ 0.707 p.u. If the overload is caused by transferred power flow, $0^{\circ}<\delta<90^{\circ}, \overline{\mathrm{OB}}$ will be larger than 70.7 percent of the rated voltage.

Based on the fault voltage model, the derivation on ranges of $\min \left|U_{M i j} \cos \varphi_{M i j}\right|$ under circumstances of interphase fault and nonfault overload has provided the theoretical foundation for the voltage criterion to be established as follows:

$$
\gamma<k_{3}
$$

where $\gamma$ characterizes the phase-to-phase faults including two-phase short circuit fault, two-phase grounding fault, and three-phase short circuit fault, and $\gamma=\min \left|U_{M i j} \cos \varphi_{M i j}\right| / U_{N}, i j=a b, b c, c a$, in which $U_{M i j}$ is the amplitude of the WAMS-measured interphase voltage represented by $\dot{U}_{M i j}$ at the location of protection; $\varphi_{M i j}=\arg \left(\dot{U}_{M i j} / \dot{I}_{M i j}\right)+90^{\circ}-\varphi_{L}$, in which $\arg \left(\dot{U}_{M i j} / \dot{I}_{M i j}\right)$ represents the WAMS-measured angle between $\dot{U}_{M i j}$ and phase-to-phase differential current at the location of protection and $\varphi_{L}$ is the positive-sequence impedance angle of the protection-located line; $U_{N}$ is the rated voltage; and $k_{3}$ cannot only be suggested as a figure between 0.669 and 0.707 when relays are designed, but also be determined in terms of the on-the-spot conditions. If (50) is satisfied, the phase-to-phase fault will be identified.

\section{Flow Transferring Identification Scheme}

4.1. Strategy of Flow Transferring Identification. The 3 ratios $\alpha$, $\beta$, and $\gamma$ derived above to reflect differences between internal fault and nonfault overload are defined as Transferred Flow Characteristic Ratio (TFCR). It is applied to the 3 criteria of the novel algorithm, as is shown in (36) and (50). The types of fault that the 3 criteria can identify are listed in Table 2 .

From Table 2, the combination of 2 current criteria can identify single-phase grounding fault, 2-phase short circuit, and most of 2-phase grounding fault. The voltage criterion can identify 3-phase short circuit, 2-phase short circuit, and 2-phase grounding fault. Therefore, the cooperation of the 3 identification criteria can be used to distinguish between all types of short circuit faults and nonfault overload to prevent the incorrect operation of backup relays in time. The logical relationship of the established identification criteria is shown in Figure 21.

According to Figure 21, after the backup protection starts, if none of the 3 criteria is satisfied, overload caused by the transferred power flow will be identified and backup protective relay tripping will be blocked. Otherwise, the internal fault will be identified and the backup protective relay will be permitted to operate.

For example, suppose that zone 3 distance protection at location of Protection 1 starts because of a short circuit fault on point $K$ in Figure 1. Flow transferring identification will be in process. TFCR will be calculated to be applied to the 
TABLE 2: The types of fault that 3 criteria can identify.

\begin{tabular}{lcccc}
\hline & Single-phase grounding & 2-phase grounding & 2-phase short circuit & 3-phase short circuit \\
\hline$\alpha>k_{1}$ and $\beta>k_{2}$ & All & Most & All & - \\
$\gamma<k_{3}$ & - & All & All & All \\
\hline
\end{tabular}

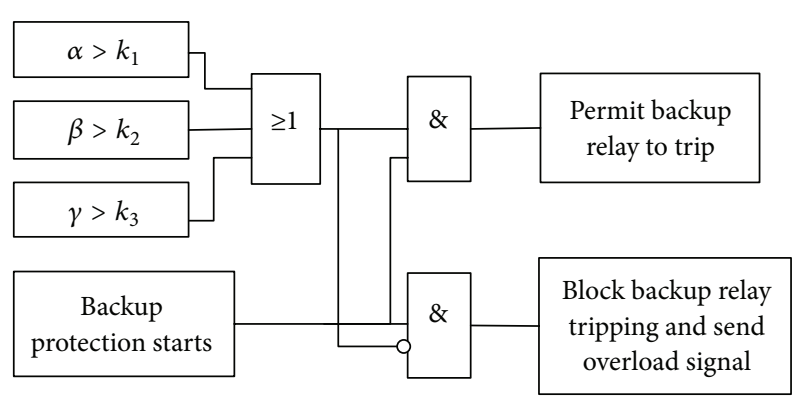

FIgURE 21: Logical relationship of the 3 criteria.

identification criteria. After the internal fault is identified, zone 3 distance relays will be permitted to trip. The relay at Protection 2 will trip Line 2 to clear the fault. What calls for special attention is that zone 3 distance relays may not reset with the clearance of fault because of the possible overload resulting from the transferred power flow on the removed Line 2 . When the operating delay is used up, zone 3 distance relays will trip the overloaded lines, deteriorating the system status. In order to avoid this situation, if any breaker trips while zone 3 distance relays do not reset, the identification process should be carried out once more. The new TFCR will be calculated to account for changing system conditions using phase current measured at Protection 1 after the breaker trips as $\dot{I}_{M \varphi}$.

Particularly, since the power swing can also make zone 3 distance protection start and can lead to identification in error, it should be detected first after zone 3 distance protection starts. If power system swings, the flow transferring identification process will not be carried out.

\subsection{Implementation Process of Power Flow Transferring Iden-} tification Algorithm. In order to realize the function of flow transferring identification, the cooperation of the regional control center and local protective devices is essential. The regional control center can be installed in an important substation or in the dispatching center. Flowcharts of regional control center and local protective devices are shown in Figures 22 and 23, respectively.

The regional control center traces the complicated network topology in real time by utilization of the WAMSmeasured information like node voltage, branch current, impedance angle, and breaker on/off state and determines which breakers are involved in the range of each zone 3 distance protection. After zone 3 distance protection starts, if power system swings, the report that power swing starts zone 3 distance protection will be sent to the control center, and the identification process will be stopped, while if the control center does not receive power swing signal, the prefault phase current $\dot{I}_{M \varphi|0|}$ will be sent to local protective devices for calculating TFCR. After an internal fault or nonfault overload is identified, the report will be sent to the control center. The regional control center will supervise the states of the breakers in the range of zone 3 distance protection which can be uploaded in 20-50 milliseconds by WAMS if an internal fault occurs. Once any breaker trips, the control center will send trip signal to local devices. If the relays do not reset, the new TFCR will be calculated to account for changing the system conditions. Otherwise, the original property of the existing zone 3 distance relays that they will operate as soon as the delay time is out under condition that the primary protective relay fails will be maintained. For zone 3 distance protection, since the operating delay is usually longer than 1 second, there is enough time to realize the flow transferring identification function.

\section{Simulation Results}

In this section, two simulation cases are applied to demonstrate the feasibility and effectiveness of the proposed algorithm. The first case is used to validate the identification reliability of the proposed criteria for types of short circuit faults via different transition resistances and at different locations as well as nonfault overload by a simple power grid. The second case is used to validate the effectiveness of the novel algorithm by IEEE 10-machine 39-node test system.

5.1. Simulation Case 1. The $500 \mathrm{kV}$ electrical power grid model with 3 nodes is constructed on MATLAB/Simulink platform, as is shown in Figure 24. Based on this model, the simulation experiments are conducted.

The power grid parameters are set as follows: $Z_{M 1}=$ $10 \angle 85.69^{\circ} \Omega$ and $Z_{N 1}=10 \angle 86.44^{\circ} \Omega$. Line 1 and Line 2 are parallel transmission lines, whose positive-sequence impedances are both $67.94 \angle 86.44^{\circ} \Omega$ and zero-sequence impedances are both $206.1 \angle 75.92^{\circ} \Omega$. The positive-sequence and zero-sequence impedances of Line 3 are $49.37 \angle 86.39^{\circ} \Omega$ and $153.8 \angle 75.78^{\circ} \Omega$, respectively. Both sides of each line are configured zone 3 distance protective devices. The highvoltage side of the transformer connected to Bus $M^{\prime}$ adopts star connection with neutral point, and the low-voltage side adopts delta connection. The equivalent impedance of the transformer is $2.5 \Omega$, and the load carried by the transformer is $(6000+j 3400)$ MVA. Set $k_{1}, k_{2}$, and $k_{3}$ as $1.778,0.795$, and 0.680 , respectively.

5.1.1. Experiment 1. Suppose that a short circuit fault on Line 3 occurs at the point which is 80 percent of the total length away from Bus $M^{\prime}$. According to Figure 23, if either of the 2 current criteria is satisfied, the voltage criterion will not be 


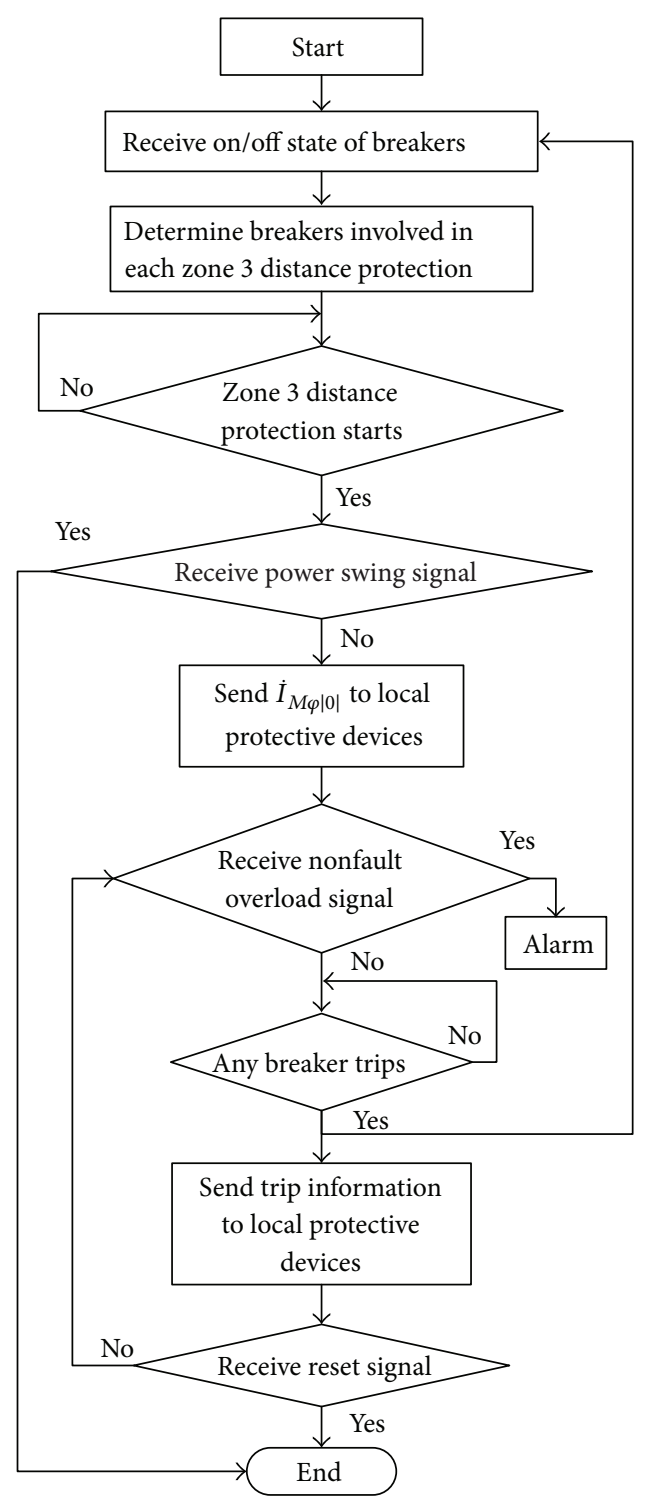

FIGURE 22: Flowchart of flow transferring identification algorithm in regional control center.

TABLE 3: TFCR under condition of single-phase grounding fault on Line 3.

\begin{tabular}{lcccccc}
\hline \multirow{2}{*}{ Protection } & \multicolumn{3}{c}{$R_{g}=0$} & \multicolumn{3}{c}{$R_{g}=100$} \\
& 1 or 3 & 5 & 6 & 1 or 3 & 5 & 6 \\
\hline$\alpha$ & 2.211 & 31.797 & 88.055 & 2.207 & 32.200 & 87.103 \\
$\beta$ & 0.095 & 0.959 & 1.011 & 0.095 & 0.962 & 1.010 \\
\hline
\end{tabular}

utilized. The values of TFCR at locations of Protection 1 or 3 , 5 , and 6 which are applied in the identification process under conditions of single-phase grounding fault, two-phase short circuit, two-phase grounding fault, and three-phase short circuit via different transition resistances are, respectively, listed in Tables 3, 4, 5, and 6, where $R_{\text {arc }}$ represents the arc resistance. $R_{g}$ represents the grounding resistance.

From the simulation results in Tables 3, 4, 5, and 6, we can find that at least one of the 3 identification criteria
TABLE 4: TFCR under condition of 2-phase short circuit fault on Line 3.

\begin{tabular}{lcccccc}
\hline \multirow{2}{*}{ Protection } & \multicolumn{3}{c}{$R_{\mathrm{arc}}=0$} & \multicolumn{3}{c}{$R_{\mathrm{arc}}=10$} \\
& 1 or 3 & 5 & 6 & 1 or 3 & 5 & 6 \\
\hline$\alpha$ & $>1000$ & $>1000$ & $>1000$ & $>1000$ & $>1000$ & $>1000$ \\
$\beta$ & 0 & 0 & 0 & 0 & 0 & 0 \\
\hline
\end{tabular}

TABLE 5: TFCR under condition of 2-phase grounding fault on Line 3.

\begin{tabular}{lcccccc}
\hline \multirow{2}{*}{ Protection } & \multicolumn{3}{c}{$R_{g}=0$} & \multicolumn{3}{c}{$R_{g}=100$} \\
& 1 or 3 & 5 & 6 & 1 or 3 & 5 & 6 \\
\hline$\alpha$ & 4.543 & 47.875 & 128.27 & 47.985 & 508.87 & 1346.9 \\
$\beta$ & 0.046 & 0.648 & 0.675 & 0.005 & 0.0617 & 0.066 \\
\hline
\end{tabular}

is satisfied under circumstances of the internal faults via different transition resistances. From Table 3, for single-phase 


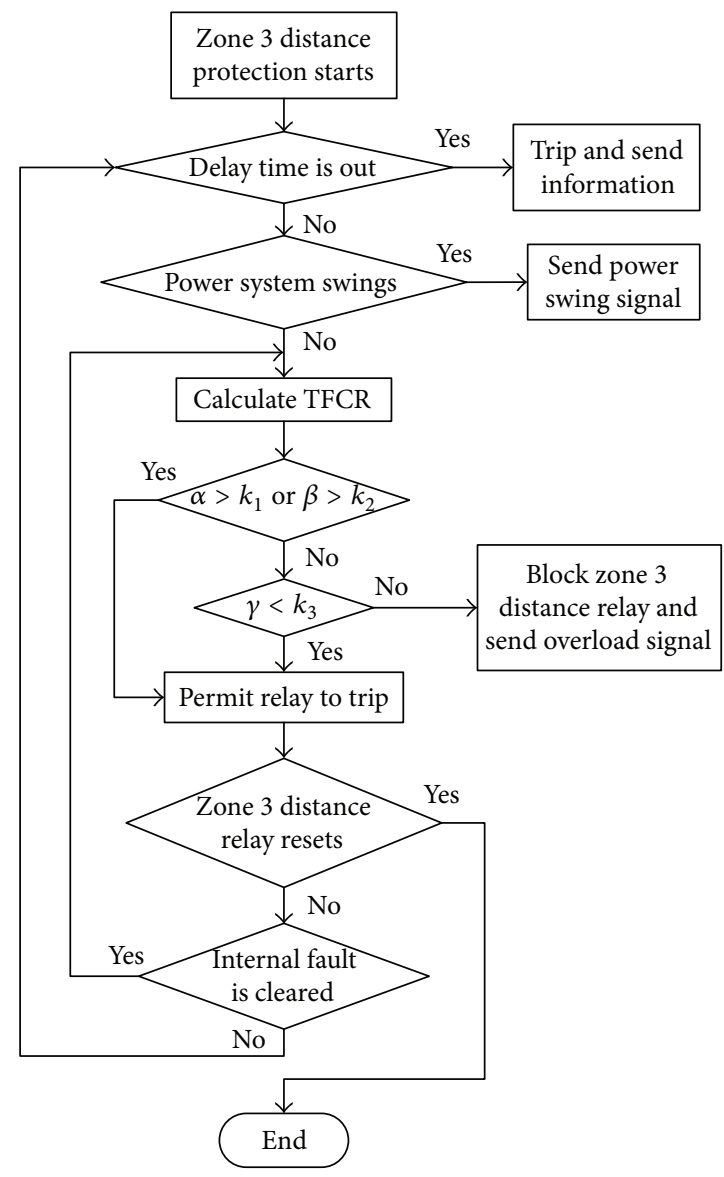

FIGURE 23: Flowchart of flow transferring identification algorithm in local protective device.

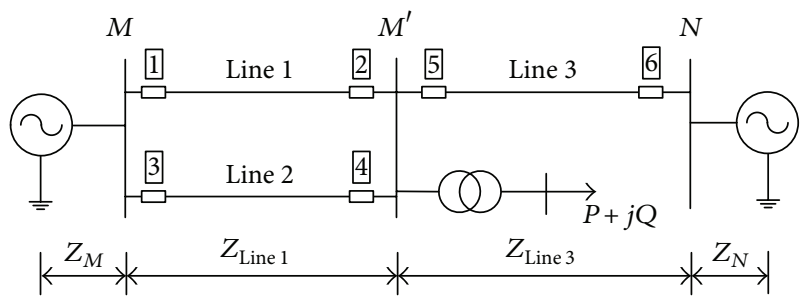

FIGURE 24: Electrical power grid for simulation experiments.

TABLE 6: TFCR under condition of 3-phase short circuit fault on Line 3.

\begin{tabular}{lcccccc}
\hline \multirow{2}{*}{ Protection } & \multicolumn{3}{c}{$R_{\mathrm{arc}}=0$} & \multicolumn{3}{c}{$R_{\mathrm{arc}}=10$} \\
& 1 or 3 & 5 & 6 & 1 or 3 & 5 & 6 \\
\hline$\alpha$ & 1.0 & 1.0 & 1.0 & 1.0 & 1.0 & 1.0 \\
$\beta$ & 0 & 0 & 0 & 0 & 0 & 0 \\
$\gamma$ & 0.1573 & 0.0051 & 0.003 & 0.3311 & 0.2629 & 0.2544 \\
\hline
\end{tabular}

grounding fault, the values of both $\alpha$ and $\beta$ are scarcely influenced by the grounding resistance. From Tables 4 and 5 , for 2-phase short circuit or 2-phase grounding fault via different transition resistances, $\alpha$ keeps far greater than 1.778 . Results in Table 6 illustrate that for 3-phase short circuit, although $\gamma$ becomes larger as the arc resistance increases, the voltage criteria will work. Thus, the sound ability of the proposed criteria to identify all types of internal short circuit faults via different transition resistances is validated.

5.1.2. Experiment 2. Suppose that a short circuit fault occurs at the end of Line 2. The values of TFCR at locations of Protection 1 or 3,2 , or 4 and 6 which are applied in the identification process under conditions of all types of short circuit faults are listed in Tables 7, 8, 9, and 10, respectively.

Similarly, the sound ability of the proposed criteria to identify the internal short circuit faults via different transition resistances is also validated by the simulation results in Tables $7,8,9$, and 10 . As is shown in Table 9, if neither of the 2 current criteria is satisfied, the voltage criterion will be utilized to identify the 2-phase grounding fault. Besides, though the location of fault in Experiment 2 is different from that in Experiment 1, faults in both experiments can be identified by the proposed criteria. In conclusion, the novel algorithm owns a high reliability to identify all types of faults at different locations as well as via different transition resistances.

5.1.3. Experiment 3. The power transmitted by Line 1 under normal operating condition is $(1108+j 313)$ MVA. Consider that the system is in state 1 . After Line 3 is removed for some 
TABLE 7: TFCR under condition of single-phase grounding fault on Line 2.

\begin{tabular}{lcccccc}
\hline \multirow{2}{*}{ Protection } & \multicolumn{3}{c}{$R_{g}=0$} & \multicolumn{3}{c}{$R_{g}=100$} \\
& 1 or 3 & 2 or 4 & 6 & 1 or 3 & 2 or 4 & 6 \\
\hline$\alpha$ & 2.301 & 18.011 & 2.210 & 2.297 & 17.997 & 2.206 \\
$\beta$ & 0.133 & 1.113 & 0.112 & 0.133 & 1.117 & 0.109 \\
\hline
\end{tabular}

TABLE 8: TFCR under condition of 2-phase short circuit fault on Line 2.

\begin{tabular}{lcccccc}
\hline \multirow{2}{*}{ Protection } & \multicolumn{3}{c}{$R_{\text {arc }}=0$} & \multicolumn{3}{c}{$R_{\text {arc }}=10$} \\
& 1 or 3 & 2 or 4 & 6 & 1 or 3 & 2 or 4 & 6 \\
\hline$\alpha$ & $>1000$ & $>1000$ & $>1000$ & $>1000$ & $>1000$ & $>1000$ \\
$\beta$ & 0 & 0 & 0 & 0 & 0 & 0 \\
\hline
\end{tabular}

TABLE 9: TFCR under condition of 2-phase grounding fault on Line 2.

\begin{tabular}{lcccccc}
\hline \multirow{2}{*}{ Protection } & \multicolumn{3}{c}{$R_{g}=0$} & \multicolumn{3}{c}{$R_{g}=100$} \\
& 1 or 3 & 2 or 4 & 6 & 1 or 3 & 2 or 4 & 6 \\
\hline$\alpha$ & 1.477 & 11.229 & 1.437 & 46.397 & 248.14 & 45.501 \\
$\beta$ & 0.211 & 1.784 & 0.171 & 0.0067 & 0.081 & 0.0054 \\
$\gamma$ & 0.0042 & & 0.0063 & & & \\
\hline
\end{tabular}

TABLE 10: TFCR under condition of 3-phase short circuit fault on Line 2.

\begin{tabular}{lcccccc}
\hline \multirow{2}{*}{ Protection } & \multicolumn{3}{c}{$R_{\mathrm{arc}}=0$} & \multicolumn{3}{c}{$R_{\mathrm{arc}}=10$} \\
& 1 or 3 & 2 or 4 & 6 & 1 or 3 & 2 or 4 & 6 \\
\hline$\alpha$ & 1.0 & 1.0 & 1.0 & 1.0 & 1.0 & 1.0 \\
$\beta$ & 0 & 0 & 0 & 0 & 0 & 0 \\
$\gamma$ & 0.0043 & 0 & 0.0063 & 0.2057 & 0.202 & 0.2050 \\
\hline
\end{tabular}

TABLE 11: Electrical quantities at Protection 1 in state 1, 2 and 3 (p.u.).

\begin{tabular}{lcccccc}
\hline State & $\dot{U}_{M i j}$ & $\dot{I}_{M i j}$ & $Z_{M i j}$ & $\alpha$ & $\beta$ & $\gamma$ \\
\hline 1 & $1.000 \angle 27.7^{\circ}$ & $0.998 \angle 12^{\circ}$ & $0.998 \angle 15.9^{\circ}$ & 1.0 & 0 & 0.93 \\
2 & $0.998 \angle 29.0^{\circ}$ & $1.258 \angle 7.3^{\circ}$ & $0.793 \angle 21.7^{\circ}$ & 1.0 & 0 & 0.89 \\
3 & $1.004 \angle 31.1^{\circ}$ & $1.697 \angle 0.3^{\circ}$ & $0.592 \angle 30.8^{\circ}$ & 1.0 & 0 & 0.81 \\
\hline
\end{tabular}

reason (such as the short circuit fault mentioned above), part of the power flow on Line 3 is transferred to Line 1 . The power transmitted by Line 1 is $(1347+j 536)$ MVA. At this time the system is considered to enter state 2 . Next, after Line 2 is removed for some reason as well, the power flow on Line 2 is transferred to Line 1 . The power transmitted by Line 1 is $(1690+j 1006)$ MVA. The system is considered to enter state 3. Obviously, the transferred power flow on the removed Line 2 and Line 3 has increased the burden of Line 1 . In the 3 states, some measured and calculated electrical quantities at Protection 1 are listed in Table 11, where $Z_{M i j}$ represents the measured impedance.

As is shown in Figure 25, the impedance measured in state 3 has encroached into zone 3 distance characteristic. The transferred power flow has resulted in the overload on Line 3. From the simulation results in Table 11, we can find that none of the 3 identification criteria is satisfied in the 3 states.

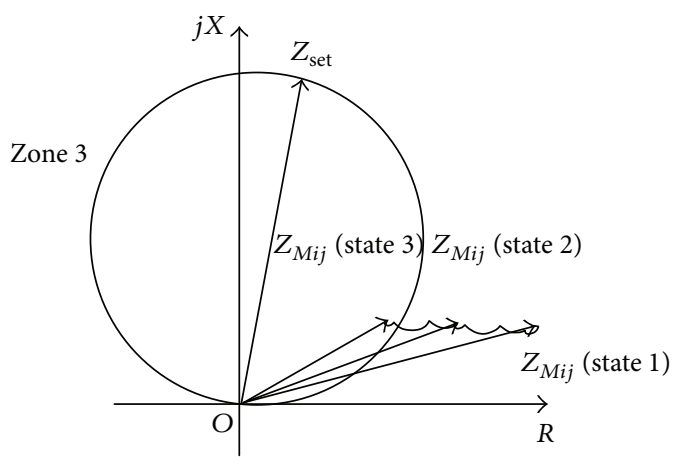

FIGURE 25: The measured impedance.

TABLE 12: TFCR under condition that an internal fault occurs near Bus 5.

\begin{tabular}{lccccccccc}
\hline \multirow{2}{*}{ Protection } & \multicolumn{10}{c}{ TFCR } \\
& $\alpha^{(1)}$ & $\beta^{(1)}$ & $\alpha^{(2)}$ & $\beta^{(2)}$ & $\alpha^{(1,1)}$ & $\beta^{(1,1)}$ & $\alpha^{(3)}$ & $\beta^{(3)}$ & $\gamma^{(3)}$ \\
\hline $\mathrm{P}_{3-4,3}$ & 5.54 & 0.64 & 6.56 & 0 & 4.12 & 0.34 & 1.0 & 0 & 0.047 \\
$\mathrm{P}_{14-4,14}$ & 13.83 & 1.22 & 4.73 & 0 & 6.29 & 0.80 & 1.0 & 0 & 0.096 \\
$\mathrm{P}_{7-6,7}$ & 2.25 & 0.46 & 5.23 & 0 & 3.38 & 0.62 & 1.0 & 0 & 0.094 \\
$\mathrm{P}_{9-8,9}$ & 2.23 & 0.26 & 4.77 & 0 & 2.74 & 0.12 & 1.0 & 0 & 0.041 \\
\hline
\end{tabular}

TABLE 13: TFCR under condition that an internal fault occurs near Bus 17.

\begin{tabular}{lccccccccc}
\hline Protection & \multicolumn{10}{c}{ TFCR } \\
& $\alpha^{(1)}$ & $\beta^{(1)}$ & $\alpha^{(2)}$ & $\beta^{(2)}$ & $\alpha^{(1,1)}$ & $\beta^{(1,1)}$ & $\alpha^{(3)}$ & $\beta^{(3)}$ & $\gamma^{(3)}$ \\
\hline $\mathrm{P}_{19-16,19}$ & 15.98 & 1.17 & 6.02 & 0 & 7.21 & 0.56 & 1.0 & 0 & 0.100 \\
$\mathrm{P}_{21-16,21}$ & 23.59 & 1.06 & 9.48 & 0 & 10.76 & 0.49 & 1.0 & 0 & 0.094 \\
$\mathrm{P}_{26-27,26}$ & 30.87 & 1.07 & 8.66 & 0 & 9.55 & 0.49 & 1.0 & 0 & 0.059 \\
$\mathrm{P}_{3-18,3}$ & 48.37 & 1.04 & 15.90 & 0 & 17.95 & 0.47 & 1.0 & 0 & 0.045 \\
\hline
\end{tabular}

Therefore, the reliability of the proposed criteria to identify the nonfault overload is validated.

5.2. Simulation Case 2. The IEEE 10-machine 39-node test system with 46 branches ( 34 lines and 12 transformers) is shown in Figure 26. The system frequency is $60 \mathrm{~Hz}$. Both sides of each line are configured zone 3 distance protective devices. Two experiments are conducted to validate the feasibility and effectiveness of the proposed algorithm.

5.2.1. Experiment 1. Two examples of the internal faults near Bus 5 and Bus 17, respectively, are given. The values of TFCR at locations of the relevant backup protections which are applied in the identification process under conditions of single-phase grounding fault, two-phase short circuit, twophase grounding fault, and three-phase short circuit are listed in Tables 12 and 13. $\mathrm{P}_{g-h, g}$ represents the protection installed near Bus $g$ on the line from Bus $g$ to Bus $h . \alpha^{(3)}, \beta^{(3)}$, and $\gamma^{(3)}$ characterize three-phase short circuit.

After the fault occurs, zone 3 distance protection will start, and the flow transferring identification will be in process. The values of TFCR shown in Tables 12 and 13 demonstrate that, under circumstances of all types of internal 


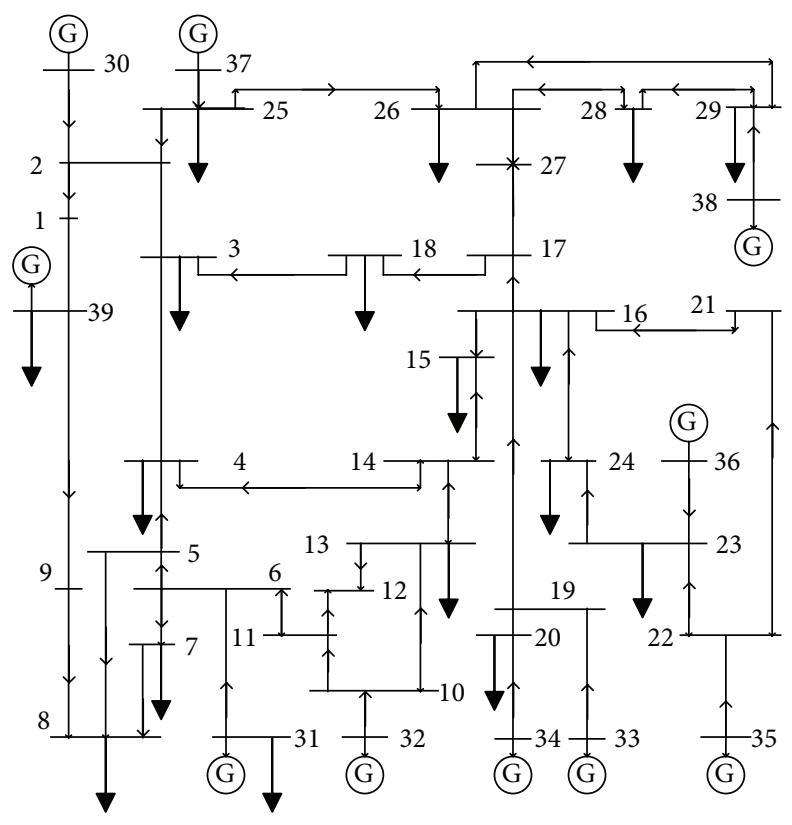

FIgURE 26: The IEEE 10-machine 39-node test system.

short circuit faults, the proposed algorithm will reliably identify the internal fault, for at least one of the 3 criteria is satisfied. Zone 3 distance relay tripping will be permitted to eliminate the internal fault in case of primary protection failure.

5.2.2. Experiment 2. Regard $l_{g-h}$ as the line from Bus $g$ to Bus $h$. Under normal operating condition, the power transmitted by $l_{14-4}$ is as much as $(262.7+j 45.5)$ MVA. The impedance measured at the location of protection on $l_{14-4}$ near Bus 14 is approximately $7.224 \angle 9.83^{\circ}$ ohms. After $l_{3-2}, l_{5-4}$, and $l_{18-17}$ are removed for some reason, the power transmitted by $l_{14-4}$ reaches $(996.9+j 470.9)$ MVA because of the additional transferred power flow on the removed lines. At this time, the impedance measured at the location of protection on $l_{14-4}$ near Bus 14 is nearly $1.396 \angle 25.28^{\circ} \mathrm{ohms}$, which has encroached zone 3 distance characteristic. After the protective devices start, if the nonfault overload on $l_{14-4}$ was not identified, $l_{14-4}$ would be removed.

Thanks to the proposed algorithm, the nonfault overload will be reliably identified. The calculation result of TFCR is as follows: $\alpha=1.0, \beta=0$, and $\gamma=0.793$. So none of the 3 identification criteria will be satisfied. According to the flowchart in Figure 23, zone 3 distance relay on $l_{14-4}$ will be blocked successfully rather than operate incorrectly.

\section{Conclusion}

In this paper, to avoid the possible incorrect operation of backup protective relays resulting from the transferred power flow, a novel algorithm for flow transferring identification based on WAMS is proposed. The criteria of the proposed algorithm adopt a new concept of TFCR which is presented and is derived mathematically by utilization of power system fault modeling. The scheme and its implementation of the novel algorithm are also provided. The feasibility and effectiveness of the proposed algorithm are demonstrated by a large number of simulations.

The proposed algorithm owns high reliability and flexibility, for its identification accuracy is not influenced by the different fault locations, the different transition resistances, and variation in reactive power infection, and the settings of criteria can not only be determined before relays are put in use but also be adjusted in terms of the on-thespot conditions. Besides, the algorithm is simple in principle and will not increase much computing and communicating burdens. Moreover, it does not change the original property of the distance protection. In conclusion, the proposed algorithm can effectively prevent the malfunction of backup relays caused by transferred power flow and can improve the performance of traditional backup protection.

\section{Conflict of Interests}

The authors declare that there is no conflict of interests regarding the publication of this paper.

\section{Acknowledgments}

This work is supported by the National Natural Science Foundation of China (Grants nos. 50777016 and 50837002) and the Fundamental Research Funds for the Central Universities (Grant no. 12MS110).

\section{References}

[1] D. Novosel, G. Bartok, G. Henneberg, P. Mysore, D. Tziouvaras, and S. Ward, "IEEE PSRC report on performance of relaying 
during wide-area stressed conditions," IEEE Transactions on Power Delivery, vol. 25, no. 1, pp. 3-17, 2010.

[2] X. Gao, K.-Q. Zhuang, and Y. Sun, "Lessons and enlightenment from blackout occurred in UCTE grid on November 4," Power System Technology, vol. 31, no. 1, pp. 25-31, 2007.

[3] A. J. Mao, G. L. Zhang, Y. C. Lü, and J. Gao, "Analysis on largescale blackout occurred in South America and North Mexico interconnected power grid on Sept. 8, 2011 and lessons for electric power dispatching in China," Power System Technology, vol. 36, no. 4, pp. 74-78, 2012.

[4] Z. Q. He, Z. Zhang, X. G. Yin, and W. Chen, "Overview of power system wide area protection," Electric Power Automation Equipment, vol. 30, no. 5, pp. 125-130, 2010.

[5] S. Xiang, Y. He, and K. Wu, "Blackout analysis of domestic power based on fractal theory," Transactions of China Electrotechnical Society, vol. 28, no. 2, pp. 367-371, 2013.

[6] The IEEE Standards Association, "IEEE guide for synchronization, calibration, testing, and installation of phasor measurement units (PMUs) for power system protection and control," IEEE Standard C37.242-2013, The IEEE Standards Association, 2013.

[7] Q. X. Yang, T. S. Bi, and J. T. Wu, "WAMS implementation in China and the challenges for bulk power system protection," in Proceedings of the IEEE Power Engineering Society General Meeting, pp. 1-6, Tempa, Fla, USA, June 2007.

[8] P. Romano and M. Paolone, "Enhanced interpolated-DFT for synchrophasor estimation in FPGAs: theory, implementation, and validation of a PMU prototype," IEEE Transactions on Instrumentation and Measurement, vol. 63, no. 12, pp. 28242836, 2014.

[9] T. S. Bi, H. Liu, D. N. Zhang, and Q. X. Yang, "The PMU dynamic performance evaluation and the comparison of PMU standards," in Proceedings of the IEEE Power and Energy Society General Meeting (PES '12), pp. 1-5, IEEE, San Diego, Calif, USA, July 2012.

[10] K.-P. Lien, C.-W. Liu, C.-S. Yu, and J.-A. Jiang, “Transmission network fault location observability with minimal PMU placement," IEEE Transactions on Power Delivery, vol. 21, no. 3, pp. 1128-1136, 2006.

[11] C. Rakpenthai, S. Premrudeepreechacharn, S. Uatrongjit, and N. R. Watson, "An optimal PMU placement method against measurement loss and branch outage," IEEE Transactions on Power Delivery, vol. 22, no. 1, pp. 101-107, 2007.

[12] X.-D. Liu, Q.-Y. Jiang, and Y.-J. Cao, "Optimal PMU placement to guarantee observability under N-1 condition," Proceedings of the Chinese Society of Electrical Engineering, vol. 29, no. 10, pp. 47-51, 2009.

[13] M. Esmaili, K. Gharani, and H. A. Shayanfar, "Redundant observability PMU placement in the presence of flow measurements considering contingencies," IEEE Transactions on Power Systems, vol. 28, no. 4, pp. 3765-3773, 2013.

[14] K. Kangvansaichol and P. A. Crossley, "Multi-zone differential protection for transmission networks," in Proceedings of 8th IEE International Conference on Developments in Power System Protection, pp. 428-431, Amsterdam, The Netherlands, April 2004.

[15] W. Cong, Z. C. Pan, J. G. Zhao, X. Z. Li, and X. P. Zhang, "A wide area protection relaying system based on current differential protection principle," Power System Technology, vol. 30, no. 5, pp. 91-110, 2006.

[16] E. E. Bernabeu, J. S. Thorp, and V. Centeno, "Methodology for a security/dependability adaptive protection scheme based on data mining," IEEE Transactions on Power Delivery, vol. 27, no. 1, pp. 104-111, 2012.

[17] J. C. Tan, P. A. Crossley, P. F. Gale, I. Hall, and J. Farrell, "Control of the central sectionaliser in a wide area back-up protection expert system," in Proceedings of the IEEE Power Engineering Society General Meeting, pp. 595-600, IEEE, Toronto, Canada, July 2003.

[18] J. C. Tan, P. A. Crossley, P. G. McLaren, I. Hall, J. Farrell, and P. Gale, "Sequential tripping strategy for a transmission network back-up protection expert system," IEEE Transactions on Power Delivery, vol. 17, no. 1, pp. 68-74, 2002.

[19] Z.-L. Yang, D.-Y. Shi, and X.-Z. Duan, "Wide-area protection system based on direction comparison principle," Proceedings of the Chinese Society of Electrical Engineering, vol. 28, no. 22, pp. 87-93, 2008.

[20] H. M. Xu, T. S. Bi, S. F. Huang, and Q. X. Yang, "WAMS based flow transfer identification algorithm," Automation of Electric Power Systems, vol. 30, no. 14, pp. 14-19, 2006.

[21] Y. D. Zhang, B. C. Chen, H. Y. Xing, C. H. Tian, and Y. Yao, "Algorithm of distance protection based on transfer power flow sensibility," Electric Power Automation Equipment, vol. 28, no. 11, pp. 35-39, 2008.

[22] H. Liu, Z. Zhou, D. Wang, and X. Wang, "Principe of distance protection capable to cope with overload," Power System Technology, vol. 38, no. 11, pp. 2943-2947, 2014.

[23] A. N. Sarwade, P. K. Katti, and J. G. Ghodekar, "Advanced distance relay characteristics suitable for dynamic loading," in Proceedings of the 9th International Power and Energy Conference (IPEC '10), pp. 509-514, Singapore, October 2010.

[24] H. K. Zadeh and Z. Li, "Adaptive load blinder for distance protection," International Journal of Electrical Power and Energy Systems, vol. 33, no. 4, pp. 861-867, 2011. 


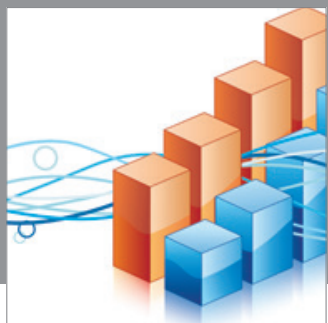

Advances in

Operations Research

mansans

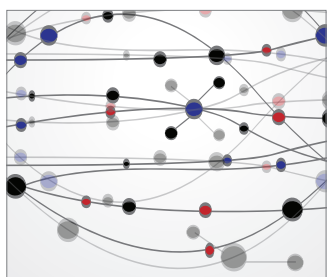

The Scientific World Journal
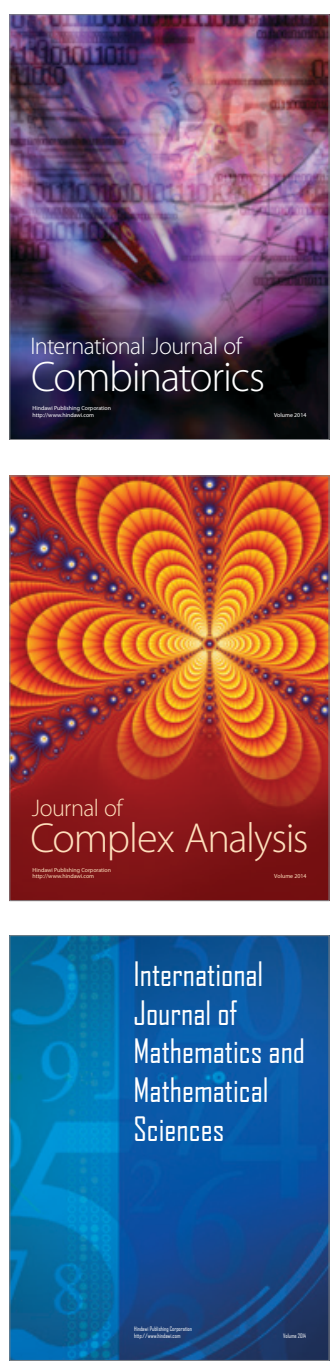
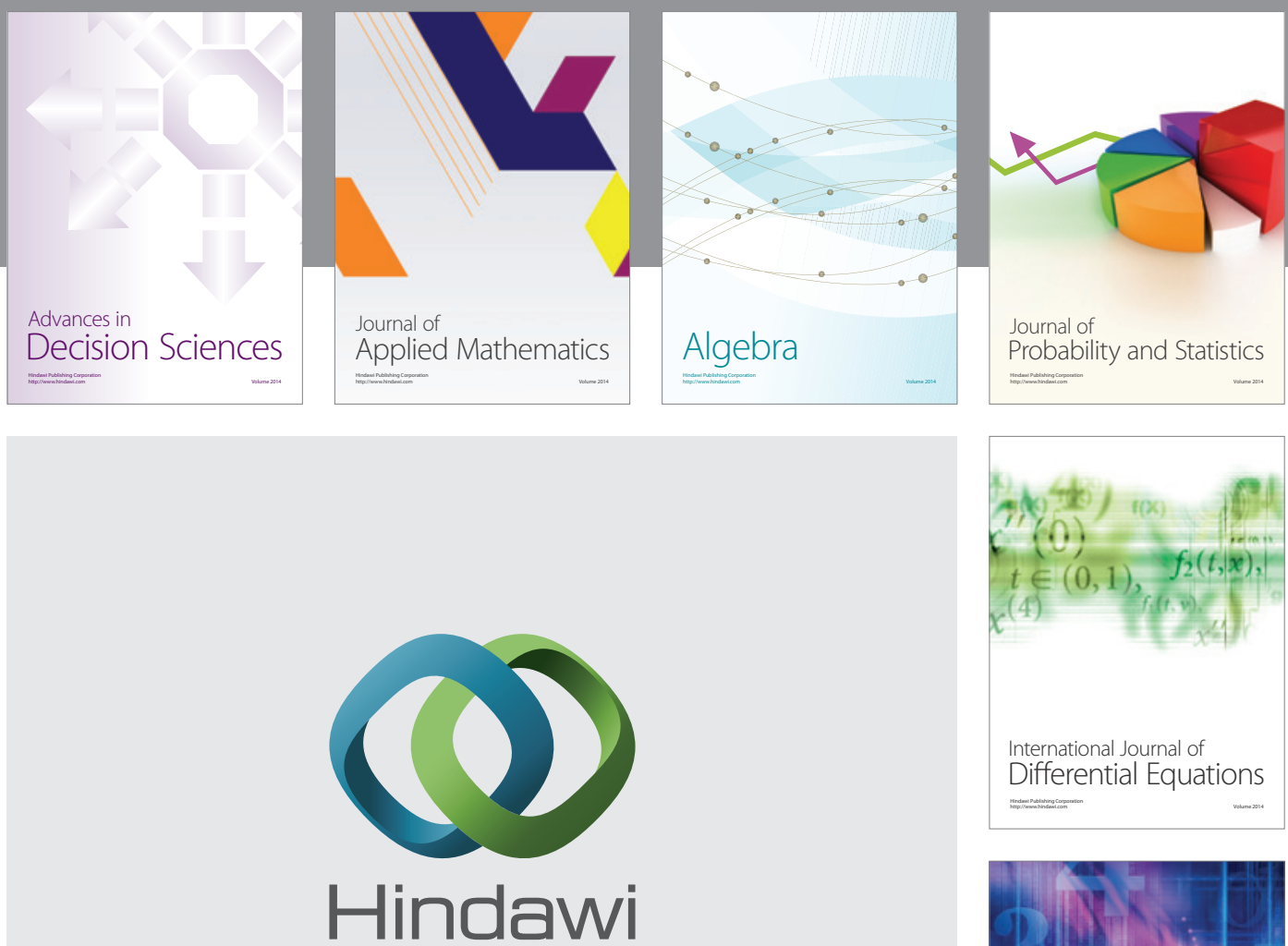

Submit your manuscripts at http://www.hindawi.com
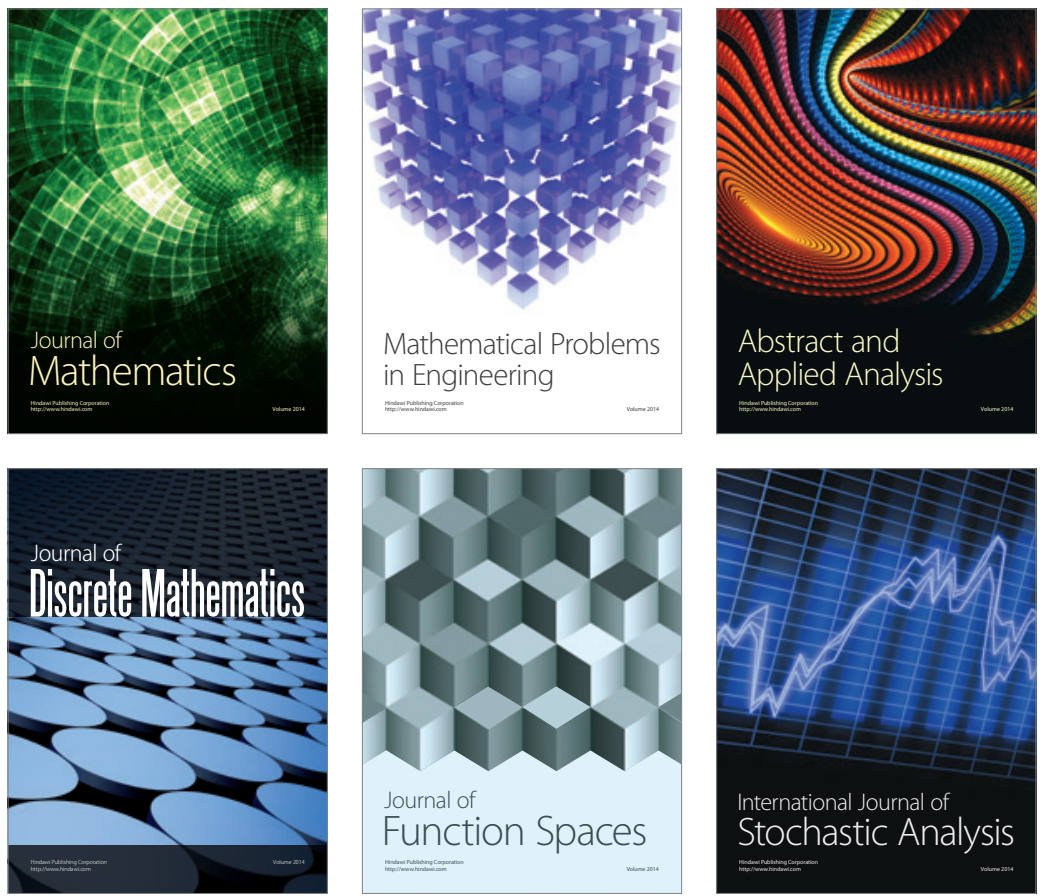

Journal of

Function Spaces

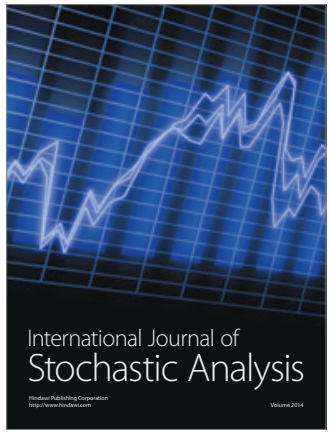

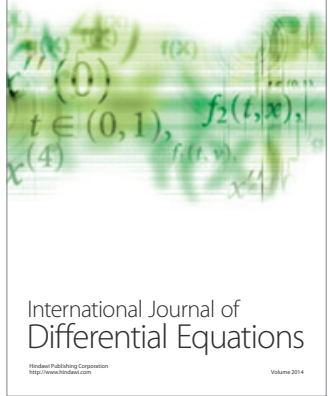
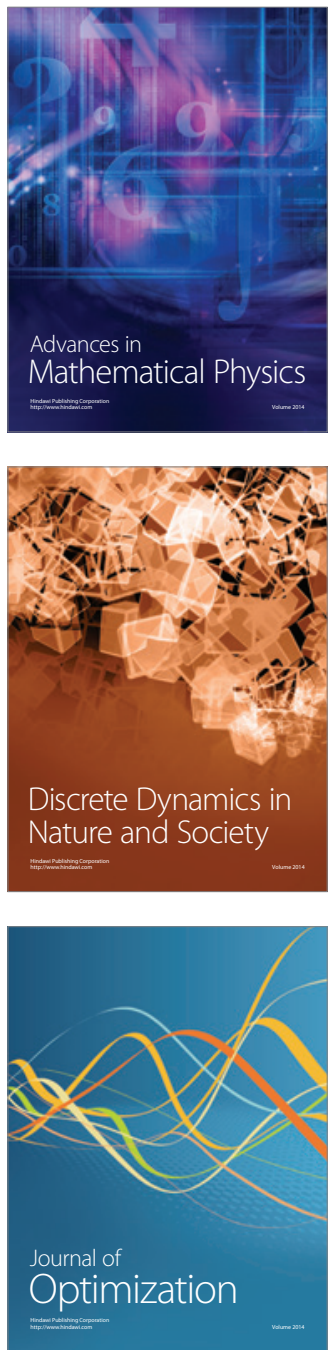